\title{
Low Conservative Criteria for Robust Consensus of Multiagent Systems with Delays, Disturbances, and Topologies Uncertainties
}

\author{
Qingjie Zhang, ${ }^{1}$ Zhongqing Jin, ${ }^{1}$ Qiang Li, ${ }^{2}$ Jianwu Tao, ${ }^{1}$ Qiongjian Fan, ${ }^{1}$ and Xiang Gao \\ ${ }^{1}$ Aviation University of Air Force, Changchun 130022, China \\ ${ }^{2}$ Changchun University of Technology, Changchun 130022, China
}

Correspondence should be addressed to Qingjie Zhang; nudtzhang@hotmail.com

Received 29 August 2013; Accepted 20 November 2013; Published 23 January 2014

Academic Editor: Yijing Wang

Copyright (C) 2014 Qingjie Zhang et al. This is an open access article distributed under the Creative Commons Attribution License, which permits unrestricted use, distribution, and reproduction in any medium, provided the original work is properly cited.

\begin{abstract}
Considering the limited communications conditions such as delays, disturbances, and topologies uncertainties, the stability criteria for robust consensus of multiagent systems are proposed in this paper. Firstly, by using the idea of state decomposition and space transformation, the condition for guaranteeing consensus is converted into verifying the robust stability of the disagreement system. In order to deal with multiple time-varying delays and switching topologies, jointly quadratic common LyapunovKrasovskii (JQCLK) functional is built to analyze the robust stability. Then, the numerical criterion can be obtained through solving the corresponding feasible nonlinear matrix inequality (NLMI); at last, nonlinear minimization is used like solving cone complementarity problem. Therefore, the linear matrix inequality (LMI) criterion is obtained, which can be solved by mathematical toolbox conveniently. In order to relax the conservativeness, free-weighting matrices (FWM) method is employed. Further, the conclusion is extended to the case of strongly connected topologies. Numerical examples and simulation results are given to demonstrate the effectiveness and the benefit on reducing conservativeness of the proposed criteria.
\end{abstract}

\section{Introduction}

Recently, the consensus problem has become an interesting and important topic in the field of formation control, flocking, rendezvous in multiagent systems, fusion estimation, collaborative decision making, and coupled oscillator synchronization.

In some practical applications, communication delays and switching topologies should be considered because of agents moving, communication congestion, or finite transmission distance. The earliest work focused on the conditions for guaranteeing that the agents achieve average consensus with time-delays and switching topologies, for example, frequency domain [1-3], time domain [4-8], delay graph [9, $10]$, and max-min value $[11,12]$. These existing methodologies contributed to the average consensus problem with limited networks enormously.

Furthermore, there is also some work focusing on the networks with disturbances, and topologies uncertainties, namely, robust consensus. The stability with noises in the multiagent systems has been discussed in [13-15]. The consensus problem of high-order agent systems is discussed in [16]. The robustness of consensus in single integrator multiagent systems to coupling delays and switching topologies is investigated $[17,18]$. Actually, more researchers are interested in solving the robust consensus problem with both delays and disturbances; see, for example, [3] in frequency domain and [19-23] in time domain. Though the analysis methodologies in frequency domain can provide sufficient and necessary conditions for consensus seeking, it is difficult to find the common or multiple Lyapunov functions in the case of switching interconnections [3]. It is only valid for fixed topology in most cases. As a result, the analysis methodologies in time domain through building Lyapunov functional become the preferred one. However, it is still sufficient conditions to achieve consensus.

This paper focuses on the improvement of conservativeness through building Lyapunov-Krasovskii functional. In 
order to obtain the lower conservativeness, free-weighting matrices (FWM) method [24-26] is adopted. This idea has been proved when solving the average consensus problem [8]. Meanwhile, this method can also relax the restrictions to the communication conditions and find the numerical criterion. Therefore, it can be employed to deal with the robust consensus with complicated network restrictions, including multiple time-varying communication delays, disturbances with limit energy, and time-varying topologies uncertainties.

The remainder of this paper is organized as follows. Section 2 contains the background and some preliminaries. Section 3 describes the problem. Main result is given in Section 4. Numerical example and simulation results are illustrated in Section 5. At last, Section 6 gives the conclusion.

Throughout this paper, the notation $\star$ represents the symmetric part in a symmetric matrix; consider $\mathscr{D}>(\geq,<$, $\leq$ )0 denotes that the matrix $\mathscr{D}$ is positive definite (positive semidefinite, negative, negative semidefinite); 0 or $\mathbf{0}$ denotes zero value or zero matrix with appropriate dimensions. $\otimes$ denotes the Kronecker product.

\section{Background and Preliminaries}

2.1. Graph Theory. Let $G=(\mathscr{V}, \mathscr{E}, \mathscr{A})$ denote the relationship between multiple agents with the set of nodes $\mathscr{V}=$ $\left\{v_{1}, \ldots, v_{n}\right\}$, the set of edges $\mathscr{E} \subset \mathscr{V} \times \mathscr{V}$, and adjacent matrix $\mathscr{A}=\left[a_{i j}\right]$. The node indices belong to a finite index set $\mathscr{I}=\{1,2, \ldots, n\}$. The edge can be depicted by $\left(v_{i}, v_{j}\right)$, and the value of $a_{i j}$ corresponds to the edge of the graph; that is, $\left(v_{i}, v_{j}\right) \in \mathscr{E} \Leftrightarrow a_{i j}>0$. The neighbors of node $v_{i}$ are defined by $\mathscr{N}_{i}=\left\{v_{j} \in \mathscr{V}:\left(v_{i}, v_{j}\right) \in \mathscr{E}\right\}$. Let $\mathbf{L}=\left[l_{i j}\right]$ denote the Laplacian matrix corresponding to the topology of the graph, where $l_{i i}=\sum_{j=1}^{n} a_{i j}$ and $l_{i j}=-a_{i j}, i \neq j$. Let $x_{i} \in R^{N_{d}}$ represent the state value of node $v_{i}$ with $N_{d}$ dimensions (such as position or temperature, etc.), and the multiagent systems with the state vector $x=\left(x_{1}, \ldots, x_{n}\right)^{T}$ and the topology $G$ can be described by $G_{x}=(G, x)$ (usually denote $G$ by compactness). We say nodes $v_{i}$ and $v_{j}$ agree in multiagent systems if and only if $x_{i}=x_{j}$. The nodes achieve consensus if and only if $x_{i}=x_{j}$ for all $i, j \in \mathscr{I}, i \neq j$. Whenever the nodes are all in agreement, the common value of all nodes is called the group decision value [1].

2.2. Several Definitions and Lemmas. Before giving the main results, we introduce some definitions and lemmas which play an important role in the proof of our main theoretical results.

Definition 1 (balanced graphs [1]). One says the node $v_{i}$ of a digraph $G=(\mathscr{V}, \mathscr{E}, \mathscr{A})$ is balanced if and only if its in-degree and out-degree equal, that is, $\operatorname{deg}_{\text {out }}\left(v_{i}\right)=\operatorname{deg}_{\text {in }}\left(v_{i}\right)$. A graph $G=(\mathscr{V}, \mathscr{E}, \mathscr{A})$ is called balanced if and only if all of nodes are balanced or $\sum_{j} a_{i j}=\sum_{j} a_{j i}, \forall i \in \mathscr{I}$.

Definition 2 (strongly connected [1]). If there is a directed path from every node to every other node, the graph is said to be strongly connected (connected for undirected graph).
Definition 3 (jointly connected [27]). By the union of a collection of simple graphs $\left\{G_{1}, G_{2}, \ldots, G_{N_{\mathrm{ss}}}\right\}$, each, with nodes set $\mathscr{V}$, is meant the simple graph $G$ with nodes set $\mathscr{V}$ and edges equaling the union of the edge sets of all of the graphs in the collection. One says that such a collection is jointly connected if the union of its members is a strongly connected graph (connected for undirected graph).

Lemma 4 (see [28]). For given symmetric matrix $\mathbf{Z}$ with the form $\mathbf{Z}=\left[\mathbf{Z}_{i j}\right], \mathbf{Z}_{11} \in R^{r \times r}, \mathbf{Z}_{12} \in R^{r \times(n-r)}$, and $\mathbf{Z}_{22} \in$ $R^{(n-r) \times(n-r)}$, then $\mathbf{Z}<0$ if and only if $\mathbf{Z}_{11}<0, \mathbf{Z}_{22}-$ $\mathbf{Z}_{21} \mathbf{Z}_{11}^{-1} \mathbf{Z}_{12}<0$ or $\mathbf{Z}_{22}<0, \mathbf{Z}_{11}-\mathbf{Z}_{12} \mathbf{Z}_{22}^{-1} \mathbf{Z}_{21}<0$.

Lemma 5 (see [29]). There exists a symmetrical matrix $\mathbf{X}$ such that the following two inequalities hold:

$$
\left[\begin{array}{cc}
\mathbf{O}_{1}+\mathbf{X} & \mathbf{Y}_{1} \\
\star & \mathbf{H}_{1}
\end{array}\right]>0, \quad\left[\begin{array}{cc}
\mathbf{O}_{2}-\mathbf{X} & \mathbf{Y}_{2} \\
\star & \mathbf{H}_{2}
\end{array}\right]>0
$$

if and only if

$$
\left[\begin{array}{ccc}
\mathbf{O}_{1}+\mathbf{O}_{2} & \mathbf{Y}_{1} & \mathbf{Y}_{2} \\
\star & \mathbf{H}_{1} & 0 \\
\star & \star & \mathbf{H}_{2}
\end{array}\right]>0
$$

Lemma 6 (see [30]). Given matrices $\mathbf{B}=\mathbf{B}^{T}$, J, K with proper dimensions, then $\mathbf{B}+\mathbf{J D}(t) \mathbf{K}+\mathbf{K}^{T} \mathbf{D}^{T}(t) \mathbf{J}^{T}<0$ holds for all $\mathbf{D}(t)$ satisfying $\mathbf{D}^{T}(t) \mathbf{D}(t) \leq \mathbf{I}$ if and only if there exists constant number $c_{0}$ such that $\mathbf{B}+c_{0}^{-1} \mathbf{J J}^{T}+c_{0} \mathbf{K}^{T} \mathbf{K}<0$ holds.

\section{Problem Description}

3.1. Agent Dynamics and Consensus Protocol. We assume that the agents are depicted by a first-order integrator dynamics, as considered in $[1,19]$. This results in a tractable mathematical problem, although some ideas can be extended to high-order or linear models. Suppose that agent $i$ can be described by

$$
\dot{\mathbf{x}}_{i}(t)=\mathbf{u}_{i}(t)+\omega_{i}(t),
$$

where $\mathbf{x}_{i}(t)$ is the state of agent $i, \mathbf{u}_{i}(t)$ is the consensus protocol and $\boldsymbol{\omega}_{i}(t)$ is the external disturbance. Moreover, $\mathbf{x}_{i}(t), \mathbf{u}_{i}(t), \boldsymbol{\omega}_{i}(t) \in \mathbb{R}^{N_{d}}$.

For the purpose of reaching robust consensus, the consensus protocol is adopted as follows:

$$
\mathbf{u}_{i}(t)=\beta_{0} \sum_{q=1}^{m} \sum_{j \in \mathcal{N}_{i}}\left(a_{i j}+\Delta a_{i j}\right)\left[\mathbf{x}_{j}\left(t-\tau_{q}\right)-\mathbf{x}_{i}\left(t-\tau_{q}\right)\right],
$$

where $\Delta a_{i j}$ denotes the uncertainties of the communication topologies. In practical terms, it may be influenced by communication congestion or the transmission distance. Parameter $\beta_{0}$ is the positive constant coefficient and $\tau_{q}, q=$ $1,2, \ldots, m$ are the time-varying communication delays.

3.2. Switching Interconnections. Consider an infinite sequence of nonempty, bounded, and contiguous timeintervals $\left[t_{k}, t_{k+1}\right), k=0,1, \ldots$, with $t_{0}=0$ and 
$t_{k+1}-t_{k} \leq T_{w \text { dell }}(k \geq 0)$ for some constant $T_{w \text { dell }}>0$. Suppose that in each interval $\left[t_{k}, t_{k+1}\right)$ there is a sequence of nonoverlapping subintervals $\left[t_{k_{0}}, t_{k_{1}}\right),\left[t_{k_{2}}, t_{k_{3}}\right), \ldots,\left[t_{k_{N_{\mathrm{ss}}-1}}, t_{k_{N_{\mathrm{ss}}}}\right)$, where $t_{k}=t_{k_{0}}$ and $t_{k+1}=t_{k_{N_{\mathrm{ss}}}}$ satisfy $t_{k_{j+1}}-t_{k_{j}} \geq T_{\text {dell }}(0 \leq$ $j<N_{\text {ss }}$ ) for some integer $N_{\text {ss }} \geq 0$ and given constant $T_{\text {dell }}>0$ such that, during each of such subintervals, the interconnection topology does not change.

Let $\sigma(t):[0, \infty] \rightarrow\left\{1,2, \ldots, N_{\mathrm{ss}}\right\}$ denote the switching signal with successive times to describe the topology switches between subintervals; then the collective dynamics of the multiagent network with $m$ communication delays and disturbances (including uncertainties and disturbances) can be depicted in matrix form

$$
\begin{gathered}
\dot{\mathbf{x}}(t)=-\beta_{0} \sum_{q=1}^{m}\left(\mathbf{L}_{\sigma q}+\Delta \mathbf{L}_{\sigma q}\right) \mathbf{x}\left(t-\tau_{q}\right)+\boldsymbol{\omega}(t), \\
\mathbf{z}(t)=\Lambda \mathbf{x}(t),
\end{gathered}
$$

where $\mathbf{x}(t) \in \mathbb{R}^{n \times 1}$ is state vector, $\boldsymbol{\omega}(t) \in \mathbb{R}^{n \times 1}$ is disturbance vector, and $\mathbf{z}(t)$ is output vector. The efficient matrix of output vector in (5) is defined as follows:

$$
\Lambda=\left[\begin{array}{ccccc}
\frac{n-1}{n} & -\frac{1}{n} & \cdots & \cdots & -\frac{1}{n} \\
-\frac{1}{n} & \frac{n-1}{n} & -\frac{1}{n} & \cdots & -\frac{1}{n} \\
\cdots & \cdots & \ddots & \cdots & \cdots \\
-\frac{1}{n} & \cdots & -\frac{1}{n} & \frac{n-1}{n} & -\frac{1}{n} \\
-\frac{1}{n} & \cdots & \cdots & -\frac{1}{n} & \frac{n-1}{n}
\end{array}\right] .
$$

3.3. Definition of Robust Consensus. Multiagent systems (5) achieve robust consensus if and only if

$$
\begin{gathered}
\lim _{t \rightarrow \infty}\left\|\mathbf{x}_{i}(t)-\mathbf{x}_{j}(t)\right\|=0, \quad \forall i, j \in \mathscr{I}, \\
\lim _{t \rightarrow \infty} \mathbf{x}_{i}(t)=\frac{1}{n} \sum_{i=1}^{n} \mathbf{x}_{i}(0), \quad \forall i \in \mathscr{I}, \\
\|\mathbf{z}\|_{2}<\gamma\|\boldsymbol{\omega}\|_{2} ;
\end{gathered}
$$

here, $\gamma>0$

Suppose that $\boldsymbol{\omega}(t) \in \mathscr{L}_{2}[0, \infty)$ and the polytopic-type uncertainties matrices for any $\sigma(t):[0, \infty] \rightarrow\left\{1,2, \ldots, N_{\mathrm{ss}}\right\}$ satisfy

$$
\Delta \mathbf{L}_{\sigma q}=\mathbf{L}_{\sigma q}^{1} \mathfrak{R}_{\sigma}(t) \mathbf{L}_{\sigma q}^{2}, \quad q=1,2, \ldots, m,
$$

where $\mathbf{L}_{\sigma q}^{1}$ and $\mathbf{L}_{\sigma q}^{2}$ are constant matrices with appropriate dimensions and $\mathfrak{R}_{\sigma}(t)$ is unknown, real, and possibly timevarying matrix with Lebesgue measurable terms satisfying $\mathfrak{Q}_{\sigma}^{T}(t) \mathfrak{Q}_{\sigma}(t) \leq \mathbf{I}$.

\section{Main Results}

In this section, we will give the delay-dependent NLMI criteria for robust consensus seeking of multiagent system including jointly connected switching topologies and strongly connected topologies. Then, nonlinear minimization is employed to deal with the NLMI criterion like solving cone complementarity problem. Lastly, the iterative LMI algorithm is given, which the max tolerant upper bounds can be obtained by Matlab LMI toolbox directly.

Before giving the main results of convergence property, we have the following assumptions:

(A1) the switching topologies of multiagent systems are jointly connected;

(A2) the switching topologies of multiagent systems are strongly connected;

(A3) its corresponding graph is balanced;

(A4) for all jointly connected topologies determined by switching signal $\sigma$, the sum of the in-degree or outdegree of all nodes is equals to each other.

Considering the jointly connected topologies, (A1), (A3), and (A4) are the precondition of multiagent systems achieve consensus and (A2), (A3), and (A4) are the precondition for strongly connected case.

\subsection{Delay-Dependent NLMI Criterion}

Theorem 7. Consider the multiagent systems with communication delays, disturbances and topologies uncertainties under the assumption that the switching topologies determined by all switching signals $\sigma:[0, \infty) \rightarrow\left\{1,2, \ldots N_{s s}\right\}$ satisfying $(A 1)$, (A3), and (A4). Given the upper bound on communication delays $\bar{\tau}_{i}$ and the upper bound on their derivative $\bar{\mu}_{i}, i=$ $1,2, \ldots, m$, if there exist common matrices $\mathbf{P}=\mathbf{P}^{T}>0, \mathbf{Q}_{q}=$ $\mathbf{Q}_{q}^{T} \geq 0, \mathbf{R}_{q}=\mathbf{R}_{q}^{T} \geq 0, q=1,2, \ldots, m$ with proper dimensions and common free-weighting matrices $\mathbf{N}_{q}=\left[\mathbf{N}_{q}^{1}, \mathbf{N}_{q}^{2}\right]^{T}, q=$ $1,2, \ldots, m$, constant number $c_{0}$ such that $\sum_{q=1}^{m} \boldsymbol{\Xi}_{\sigma q}<0, \forall \sigma$ : $[0, \infty] \rightarrow\left\{1,2, \ldots, N_{s s}\right\}$ holds.

Where

$$
\Xi_{\sigma q}=\left[\begin{array}{ccccccccc}
\Xi_{\sigma q}^{11} & \Xi_{\sigma q}^{12} & \mathbf{P} & 0 & \Xi_{\sigma q}^{15} & \bar{\tau}_{q} \Lambda^{T} & \Xi_{\sigma q}^{17} & 0 \\
\star & \Xi_{\sigma q}^{22} & 0 & \Xi_{\sigma q}^{24} & \Xi_{\sigma q}^{25} & 0 & 0 & \Xi_{\sigma q}^{28} \\
\star & \star & -\gamma^{2} \mathbf{I} & \bar{\tau}_{q} & 0 & 0 & 0 & 0 \\
\star & \star & \star & \Xi_{\sigma q}^{44} & 0 & 0 & 0 & 0 \\
\star & \star & \star & \star & \Xi_{\sigma q}^{55} & 0 & \Xi_{\sigma q}^{57} & 0 \\
\star & \star & \star & \star & \star & -\mathbf{I} & 0 & 0 \\
\star & \star & \star & \star & \star & \star & -c_{0} \mathbf{I} & 0 \\
\star & \star & \star & \star & \star & \star & \star & -c_{0} \mathbf{I}
\end{array}\right]
$$




$$
\begin{gathered}
\Xi_{\sigma q}^{11}=\mathbf{Q}_{q}+\mathbf{N}_{q}^{1}+\left[\mathbf{N}_{q}^{1}\right]^{T}, \\
\Xi_{\sigma q}^{12}=-\beta_{0} \mathbf{P} \tilde{\mathbf{L}}_{\sigma q}-\mathbf{N}_{q}^{1}+\left[\mathbf{N}_{q}^{2}\right]^{T}, \\
\Xi_{\sigma q}^{15}=\bar{\tau}_{q} \mathbf{N}_{q}^{1}, \quad \Xi_{\sigma q}^{17}=-\beta_{0} \mathbf{P} \mathbf{L}_{\sigma q}^{1}, \\
\Xi_{\sigma q}^{22}=\left(\mu_{q}-1\right) \mathbf{Q}_{q}-\mathbf{N}_{q}^{2}-\left[\mathbf{N}_{q}^{2}\right]^{T}, \\
\Xi_{\sigma q}^{24}=-\beta_{0} \bar{\tau}_{q} \mathbf{L}_{\sigma q}, \quad \Xi_{\sigma q}^{25}=\bar{\tau}_{q} \mathbf{N}_{q}^{2}, \quad \Xi_{\sigma q}^{28}=-\beta_{0} c_{0}\left[\mathbf{L}_{\sigma q}^{2}\right]^{T}, \\
\Xi_{\sigma q}^{44}=-\bar{\tau}_{q} \mathbf{R}_{q}^{-1}, \quad \Xi_{\sigma q}^{55}=-\bar{\tau}_{q} \mathbf{R}_{q}, \quad \Xi_{\sigma q}^{57}=-\beta_{0} \bar{\tau}_{q} \mathbf{L}_{\sigma q}^{1}, \\
\widetilde{\mathbf{L}}_{\sigma q}=\mathbf{E}_{c \kappa}^{T} \mathbf{L}_{\sigma q} \mathbf{E}_{c \kappa}, \quad \widetilde{\Lambda}_{c}=\mathbf{E}_{c \kappa}^{T} \Lambda \mathbf{E}_{c \kappa}, \\
\widetilde{\mathbf{L}}_{\sigma q}^{1}=\mathbf{E}_{c \kappa}^{T} \mathbf{L}_{\sigma q}^{1} \mathbf{E}_{c \kappa}, \quad \widetilde{\mathbf{L}}_{\sigma q}^{2}=\mathbf{E}_{c \kappa}^{T} \mathbf{L}_{\sigma q}^{2} \mathbf{E}_{c \kappa} ;
\end{gathered}
$$

then the protocol (4) globally asymptotically solves the robust consensus of multiagent systems (5) satisfying the specified $H_{\infty}$ index $\gamma$.

Proof. See the Appendix.

The above conclusion derived from Theorem 7 is also valid for the strongly connected topologies case. We omit the detailed proof procedure because it is similar to that in Theorem 7.

Corollary 8. Consider the multiagent systems with communication delays, disturbances, and topologies uncertainties under the assumption that the switching topologies determined by all switching signals $\sigma:[0, \infty) \rightarrow\left\{1,2, \ldots, N_{s s}\right\}$ satisfying (A2) (A4). Given the upper bound on communication delays $\bar{\tau}_{i}$ and the upper bound on their derivative $\bar{\mu}_{i}, i=$ $1,2, \ldots, m$, if there exist common matrices $\mathbf{P}=\mathbf{P}^{T}>0$, $\mathbf{Q}_{q}=\mathbf{Q}_{q}^{T} \geq 0, \quad \mathbf{R}_{q}=\mathbf{R}_{q}^{T} \geq 0, q=1,2, \ldots, m$ with proper dimensions and common free-weighting matrices $\mathbf{N}_{q}=\left[\mathbf{N}_{q}^{1}, \mathbf{N}_{q}^{2}\right]^{T}, q=1,2, \ldots, m$, constant number $c_{0}$ such that $\sum_{q=1}^{m} \boldsymbol{\Xi}_{\sigma q}<0, \forall \sigma:[0, \infty] \rightarrow\left\{1,2, \ldots, N_{s s}\right\}$ holds; then the protocol (4) globally asymptotically solves the robust consensus of multiagent systems (5) satisfying the specified $H_{\infty}$ index $\gamma$.

Remark 9. The definition of $\boldsymbol{\Xi}_{\sigma q}$ in Corollary 8 is similar to that in Theorem 7.

Remark 10. If the feasibility of the NLMI $\sum_{q=1}^{m} \Xi_{\sigma q}<0$ exists, then the derivative of the Lyapunov-Krasovskii functional is negative definite. Therefore, the systems have robust stability. In addition, the bound condition of communication can be obtained using mathematical package. Since the existence of the term $-\bar{\tau}_{q} \mathbf{R}_{q}^{-1}, q=1,2, \ldots, m$, the allowable solution of NLMI $\sum_{q=1}^{m} \boldsymbol{\Xi}_{\sigma q}<0$ cannot be obtained by LMI toolbox directly.
4.2. Nonlinear Minimization. Defining new matrices $\Upsilon_{q}(q=$ $1,2, \ldots, m)$ with proper dimension and replacing $\mathbf{R}_{q}^{-1}$ in $\boldsymbol{\Xi}_{\sigma q}$, denote as $\widehat{\Xi}_{q}$, we have

$$
\widehat{\Xi}_{\sigma q}=\left[\begin{array}{cccccccc}
\Xi_{\sigma q}^{11} & \Xi_{\sigma q}^{12} & \mathbf{P} & 0 & \Xi_{\sigma q}^{15} & \bar{\tau}_{q} \Lambda^{T} & \Xi_{\sigma q}^{17} & 0 \\
\star & \Xi_{\sigma q}^{22} & 0 & \Xi_{\sigma q}^{24} & \Xi_{\sigma q}^{25} & 0 & 0 & \Xi_{\sigma q}^{28} \\
\star & \star & -\gamma^{2} \mathbf{I} & \bar{\tau}_{q} & 0 & 0 & 0 & 0 \\
\star & \star & \star & -\bar{\tau}_{q} \Upsilon_{q} & 0 & 0 & 0 & 0 \\
\star & \star & \star & \star & \Xi_{\sigma q}^{55} & 0 & \Xi_{\sigma q}^{57} & 0 \\
\star & \star & \star & \star & \star & -\mathbf{I} & 0 & 0 \\
\star & \star & \star & \star & \star & \star & -c_{0} \mathbf{I} & 0 \\
\star & \star & \star & \star & \star & \star & \star & -c_{0} \mathbf{I}
\end{array}\right] .
$$

Consequently,

$$
\mathbf{\Xi}_{\sigma q}<0 \sim \widehat{\Xi}_{\sigma q}<0, \quad \mathbf{R}_{q}^{-1} \geq \Upsilon_{q} .
$$

According to Lemma 4,

$$
\mathbf{R}_{q}^{-1} \geq \mathbf{Y}_{q} \sim\left[\begin{array}{cc}
\mathbf{Y}_{q}^{-1} & \mathbf{I} \\
\star & \mathbf{R}_{q}^{-1}
\end{array}\right] \geq 0 .
$$

As a result, $\boldsymbol{\Xi}_{\sigma q}<0$ if and only if $\widehat{\boldsymbol{\Xi}}_{\sigma q}<0,\left[\begin{array}{cc}\mathbf{Y}_{q}^{\prime} & \mathbf{I} \\ \star & \mathbf{R}_{q}^{\prime}\end{array}\right] \geq 0, \mathbf{\Upsilon}_{q}^{\prime}=$ $\mathbf{Y}_{q}^{-1}$ and $\mathbf{R}_{q}^{\prime}=\mathbf{R}_{q}^{-1}$ holds.

The above problem can be solved as the cone complementarity problem (CCP) in [31]:

$$
\begin{aligned}
& \min \quad \operatorname{Tr}\left\{\sum_{q=1}^{m}\left(\mathbf{Y}_{q} \mathbf{Y}_{q}^{\prime}+\mathbf{R}_{q}^{\prime} \mathbf{R}_{q}\right)\right\} \\
& \text { s.t. } \quad \sum_{q=1}^{m} \widehat{\Xi}_{\sigma q}<0, \quad \mathbf{\Upsilon}_{q}>0, \quad \sum_{q=1}^{m}\left[\begin{array}{cc}
\mathbf{Y}_{q}^{\prime} & \mathbf{I} \\
\star & \mathbf{R}_{q}^{\prime}
\end{array}\right] \geq 0, \\
& \sum_{q=1}^{m}\left[\begin{array}{cc}
\mathbf{Y}_{q} & \mathbf{I} \\
\star & \mathbf{\Upsilon}_{q}^{\prime}
\end{array}\right] \geq 0, \quad \sum_{q=1}^{m}\left[\begin{array}{cc}
\mathbf{R}_{q} & \mathbf{I} \\
\star & \mathbf{R}_{q}^{\prime}
\end{array}\right] \geq 0, \quad q=1,2, \ldots, m .
\end{aligned}
$$

Here, sign "Tr" denotes the trace of matrix.

Next, the iterative LMI algorithm is proposed to solve the problem (13). Therefore, Theorem 7 and Corollary 8 can be solved directly by the existing LMI-based methods or mathematical software package.

4.3. Iterative LMI Algorithm. There are $m$ delays between multiagent systems. For simplicity, suppose that multiple delays satisfy $\tau_{1}<\cdots<\tau_{m}$. 
Algorithm 11 (maximum of the upper bound on network delay $\tau_{m}$ with specified $H_{\infty}$ index $\gamma$ ).

Step 1. Set the initial value of $\tau_{m}$ such that $\sum_{q=1}^{m} \widehat{\boldsymbol{\Xi}}_{\sigma q}<0$.

Step 2. Find the feasible solution of four matrices variables in (13), namely, $\mathbf{\Upsilon}_{q}^{\prime}(0), \mathbf{\Upsilon}_{q}(0), \mathbf{R}_{q}^{\prime}(0), \mathbf{R}_{q}(0), q=1, \ldots, m$. Let the iterative number $k=0$.

Step 3. Solve the following minimum problem about matrices variables $\mathbf{Y}_{q}^{\prime}, \mathbf{Y}_{q}, \mathbf{R}_{q}^{\prime}, \mathbf{R}_{q}$ satisfying the restriction in (13):

$$
\begin{aligned}
\min \left\{\operatorname{Tr} \sum_{q=1}^{m}\left(\Upsilon_{q}(k) \Upsilon_{q}^{\prime}+\Upsilon_{q} \Upsilon_{q}^{\prime}(k)\right)\right. \\
\left.+\operatorname{Tr} \sum_{q=1}^{m}\left(\mathbf{R}_{q}^{\prime}(k) \mathbf{R}_{q}+\mathbf{R}_{q}^{\prime} \mathbf{R}_{q}(k)\right)\right\} .
\end{aligned}
$$

Let $\mathbf{Y}_{q}^{\prime}(k+1)=\mathbf{Y}_{q}^{\prime}, \mathbf{\Upsilon}_{q}(k+1)=\mathbf{Y}_{q}, \mathbf{R}_{q}^{\prime}(k+1)=\mathbf{R}_{q}^{\prime}$ and $\mathbf{R}_{q}(k+1)=\mathbf{R}_{q}, \forall q=1, \ldots, m$.

Step 4. Justify $\sum_{q=1}^{m} \boldsymbol{\Xi}_{\sigma q}<0$ or not. If yes, increase $\tau_{m}$ and return to Step 2; otherwise, check up the iterative time. If it exceeds the specified number, terminate the algorithm; otherwise, let $k=k+1$ and return to Step 3 .

Remark 12. As the literature [31] pointed, it is difficult to get the global solution of the nonlinear minimum problem (13). In order to get suboptimal maximum upper bound on delay, the inequality $\sum_{q=1}^{m} \Xi_{\sigma q}<0$ is adopted in the iterative algorithm.

Remark 13. Similarly, the above iterative algorithm also can be used to get the maximum upper bound on delay derived from Corollary 8. Moreover, the minimum of the index $\gamma$ with specified the upper bound the minimum of the index $\gamma$, with specified the delay, can be obtained by the iterative algorithm.

\section{Example and Discussions}

5.1. Illustrative Example. We now offer an illustrative example to show the effectiveness of the proposed method. Consider the jointly connected and balanced topologies with two communication delays; see Figure 1. For simplicity, suppose that their adjacency matrices are limited to 0 and 1 matrices. In the following, two cases with different connectivities are discussed, respectively.

Assume that the time-varying communication delays satisfy $\bar{\tau}_{1}<\bar{\tau}_{2}$ and let

$$
\begin{array}{ll}
\mathbf{L}_{a 1}^{1}=0.2 * \mathbf{I}, & \mathbf{L}_{a 1}^{2}=\mathbf{L}_{a}, \quad \mathbf{L}_{a 2}^{1}=\mathbf{L}_{a 2}^{2}=0 \\
\mathbf{L}_{b 1}^{1}=\mathbf{L}_{b 1}^{2}=0, & \mathbf{L}_{b 2}^{1}=0.2 * \mathbf{I}, \quad \mathbf{L}_{b 2}^{2}=\mathbf{L}_{b}, \\
\mathbf{L}_{c 1}^{1}=0.2 * \mathbf{I}, & \mathbf{L}_{c 1}^{2}=\mathbf{L}_{c}, \quad \mathbf{L}_{c 2}^{1}=\mathbf{L}_{c 2}^{2}=0 \\
\mathbf{L}_{d 1}^{1}=\mathbf{L}_{d 1}^{2}=0, & \mathbf{L}_{d 2}^{1}=0.2 * \mathbf{I}, \quad \mathbf{L}_{d 2}^{2}=\mathbf{L}_{d} .
\end{array}
$$

For given index $\gamma=1$, the allowable upper bound on communication delay can be obtained by Theorem 7 and

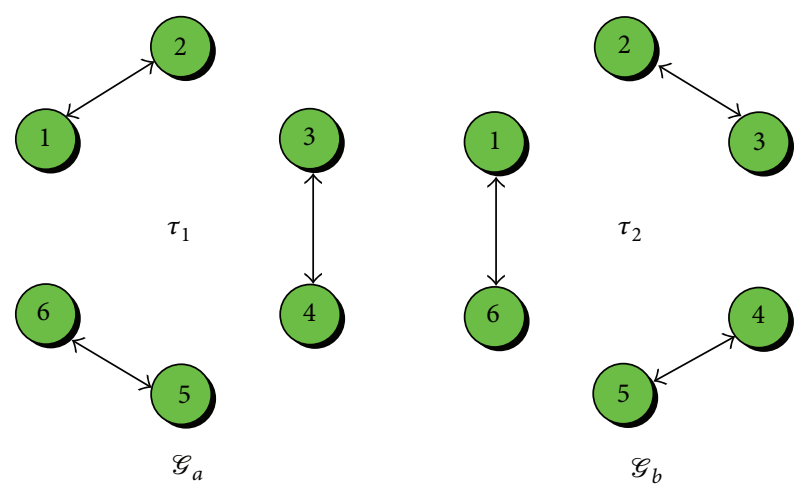

Case I

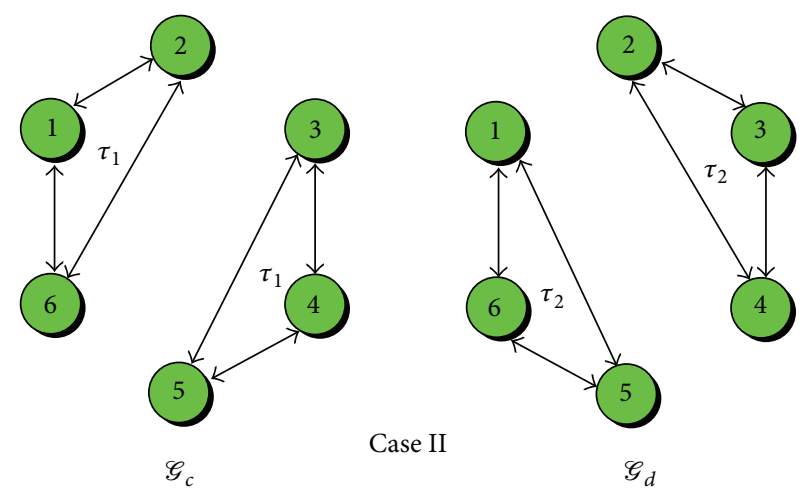

FIGURE 1: Jointly connected topologies $\mathscr{G}_{a}, \mathscr{G}_{b}$ and $\mathscr{G}_{c}, \mathscr{G}_{d}$.

Algorithm 11. In Table 1, four-factor three-level orthogonal experiment results are given by using Matlab LMI toolbox.

Remark 14. The major factor affecting $\bar{\tau}_{2}$ is the positive constant coefficient $\beta_{0}$. Then the second one is $\bar{\tau}_{1}$. However, their derivatives $\bar{\mu}_{1}, \bar{\mu}_{2}$ seem not to affect the results unduly; so we can increase $\beta_{0}$ or reduce $\bar{\tau}_{1}$ to get bigger $\bar{\tau}_{2}$. This also describing the relationship between each factor and the upper bound on communication delay.

Remark 15. Since the delay derivative has little effect on the communication delay, we can obtain the delaydependent/derivative-independent criterion through excluding the second item in (A.4). Plenty of experiments show that their numerical results are basically the same.

Remark 16. The allowable upper bound on communication delays for Case II is bigger than Case I. That is because Case II has higher connection property, which is measured by the second smallest eigenvalue of the Laplacian matrix for jointly connected topologies.

Remark 17. The proposed criteria for robust consensus seeking can be employed to maximize $\bar{\tau}_{2}$ for different $\bar{\mu}_{2}, \bar{\tau}_{1}$, and $\bar{\mu}_{1}$ and $\beta_{0}$ with specified $\gamma$; on the other hand, they also can be used to minimize $\gamma$ for given $\bar{\tau}_{1}, \bar{\mu}_{1}, \bar{\tau}_{2}, \bar{\mu}_{2}$, and $\beta_{0}$. 
TABLE 1: Four-factor three-level orthogonal experiment.

\begin{tabular}{|c|c|c|c|c|c|c|}
\hline \multirow{2}{*}{ No. } & \multicolumn{4}{|c|}{ Factor } & \multicolumn{2}{|c|}{ Index value $\bar{\tau}_{2}$} \\
\hline & $\beta_{0}$ & $\bar{\tau}_{1}$ & $\bar{\mu}_{1}$ & $\bar{\mu}_{2}$ & Case I & Case II \\
\hline 1 & 1 & 0.05 & 0 & 0 & 0.209 & 1.279 \\
\hline 2 & 1 & 0.10 & 0.50 & 0.50 & 0.159 & 1.229 \\
\hline 3 & 1 & 0.12 & 0.90 & 0.90 & 0.139 & 1.209 \\
\hline 4 & 2 & 0.05 & 0.50 & 0.90 & 0.987 & 5.267 \\
\hline 5 & 2 & 0.10 & 0.90 & 0 & 0.937 & 5.217 \\
\hline 6 & 2 & 0.12 & 0 & 0.50 & 0.917 & 5.197 \\
\hline 7 & 3 & 0.05 & 0.90 & 0.50 & 2.284 & 11.913 \\
\hline 8 & 3 & 0.10 & 0 & 0.90 & 2.234 & 11.864 \\
\hline 9 & 3 & 0.12 & 0.50 & 0 & 2.214 & 11.844 \\
\hline $\bar{I}_{1}$ & 0.169 & 1.160 & 1.120 & 1.120 & & \\
\hline $\bar{I}_{2}$ & 0.947 & 1.110 & 1.120 & 1.120 & & \\
\hline $\bar{I}_{3}$ & 2.244 & 1.090 & 1.120 & 1.120 & & \\
\hline Extreme err. & 2.075 & 0.070 & 0 & 0 & & \\
\hline
\end{tabular}

5.2. Simulations Results. In order to demonstrate the effectiveness of our results and the conclusion aforementioned, several simulations are given in the following.

Let

$$
\begin{aligned}
& \mathfrak{Q}_{a}(t)=\mathfrak{Q}_{c}(t)=\left[\begin{array}{cc}
\sin (t) & \mathbf{0}_{1 \times 5} \\
\mathbf{0}_{5 \times 1} & \mathbf{0}_{5 \times 5}
\end{array}\right], \\
& \mathfrak{Q}_{b}(t)=\mathfrak{Q}_{d}(t)=\left[\begin{array}{cc}
\cos (t) & \mathbf{0}_{1 \times 5} \\
\mathbf{0}_{5 \times 1} & \mathbf{0}_{5 \times 5}
\end{array}\right]
\end{aligned}
$$

denote the unknown, real, and time-varying matrix satisfying

$$
\begin{array}{ll}
\mathfrak{Q}_{a}{ }^{T}(t) \mathfrak{Q}_{a}(t) \leq \mathbf{I}, & \mathfrak{Q}_{b}{ }^{T}(t) \mathfrak{Q}_{b}(t) \leq \mathbf{I}, \\
\mathfrak{Q}_{c}{ }^{T}(t) \mathfrak{Q}_{c}(t) \leq \mathbf{I}, & \mathfrak{Q}_{d}{ }^{T}(t) \mathfrak{Q}_{d}(t) \leq \mathbf{I} .
\end{array}
$$

Select the impulse disturbance like $[19,20]$

$$
\boldsymbol{\omega}(t)= \begin{cases}0, & t \in[1,21) \\ 1, & t \in[21,22) \\ 0, & t \in[22,40] .\end{cases}
$$

For Case I, the switching signal is $\sigma(t)=\mathscr{G}_{a}$ when $t \in$ $[k T, k T+1)$, and $\sigma(t)=\mathscr{G}_{b}$ when $t \in[k T+1, k T+2)$; similarly, the switching signal is $\sigma(t)=\mathscr{G}_{c}$ when $t \in[k T, k T+1)$; and $\sigma(t)=\mathscr{G}_{d}$ when $t \in[k T+1, k T+2)$ for Case II. Here, $T=1 \mathrm{~s}$.

With the above conditions, the sixth group in Table 1 is selected. Further, we select the delay $\tau_{2}(t)=0.917|\sin ((0.5 / 0.917) t)| \mathrm{s}$ for Case I and delay $\tau_{2}(t)=5.197|\sin ((0.5 / 5.197) t)| \mathrm{s}$ for Case II. Corresponding simulation experiments are given in Figures 2(a) and 2(b) for different cases. It is clear from Figure 2 that multiagent systems achieving robust consensus with multiple delays, impulse disturbances, and time-varying topologies uncertainties. The simulation results further confirm the effectiveness of the proposed theory.

In Figures 3 and 4, the state evolvement of multiagent systems with different $\tau_{1}(t)$ and $\beta_{0}$ is given, respectively. Comparing Figure 3 with Figure 2(a), we can find out that
TABLE 2: Allowable upper bound on communication delay for firstorder multi-agent systems with fixed topology $G_{e}$ when $\gamma=2$.

\begin{tabular}{lcccc}
\hline $\bar{\mu}$ & 0 & 0.5 & 0.9 & Any \\
\hline$[19]$ Theorem 3 & 0.083 & - & - & - \\
Corollary 8 & 4.393 & 4.392 & 4.393 & 4.393 \\
\hline
\end{tabular}

the vibration of multiagent systems achieving consensus is acute and getting worse when increasing $\tau_{1}(t)$ gradually. Particularly, multiagent systems even cannot achieve robust consensus from Figure 3(c). In addition, the similar conclusion can be obtained when decreasing $\beta_{0}$; see Figures 2 (b) and 4 . These simulation results show the correctness of Remark 14.

5.3. Conservativeness Comparisons. In order to show the benefit of our results, we will compare the conservativeness of criteria with the existing results for strongly connected case [19]. Assume that there is a uniform communication delay in multiagent systems $\left(n=15, m=1\right.$, and $\left.\beta_{0}=1\right)$, and the topology can be seen in Figure 5.

Table 2 lists the allowable upper bounds on communication delay for different $\bar{\mu}$ when $\gamma=2$. The comparison result shows that the criterion derived from this paper is better than [19]. Moreover, the upper bound on the time-varying delay can be achieved with the proposed method, while the result in [19] is only valid for invariant delay. The major reason is the FWM approach which does not magnify the derivative of Lyapunov-krasovskii functional excessively.

\section{Conclusions}

The numerical criteria for robust consensus of multiagent systems were provided in this paper. It can be used to justify the convergence property of multiagent systems with multiple time-varying communication delays, disturbances with limit energy, and time-varying topologies uncertainties. Mainly, the following contributions were concluded in this paper. 


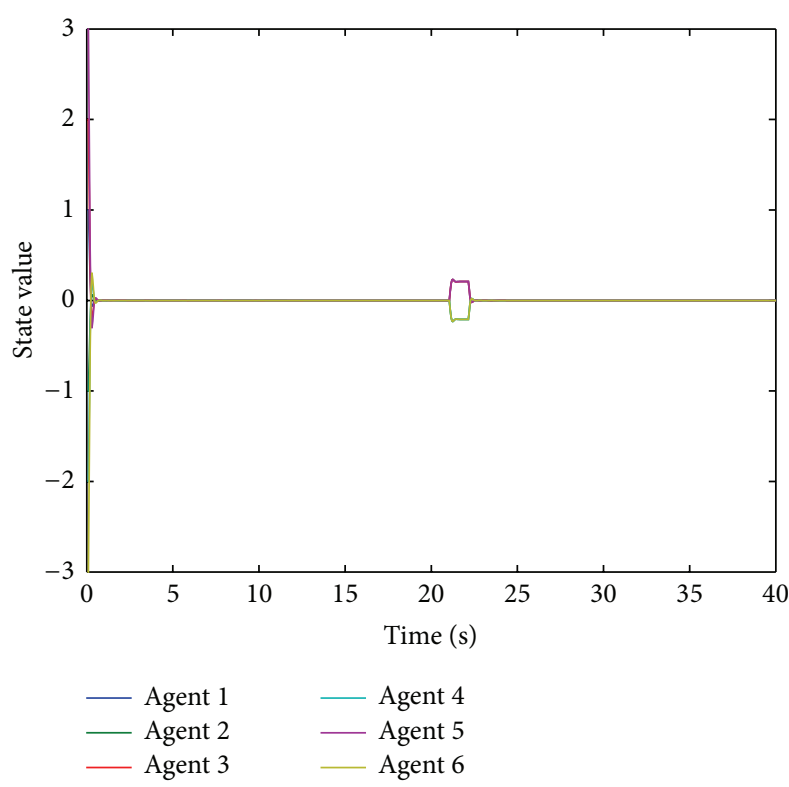

(a) Case I

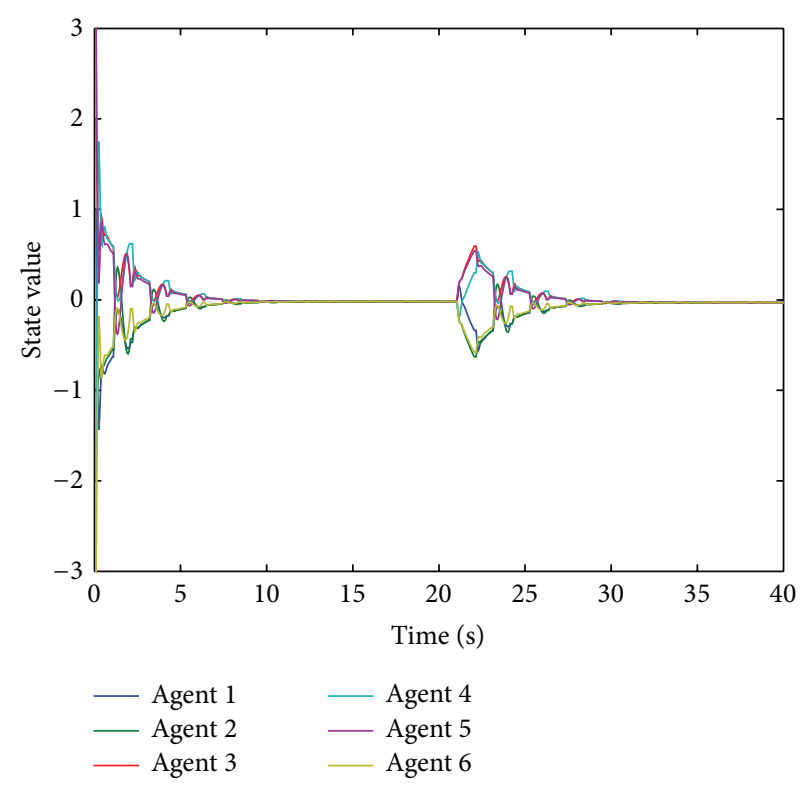

(b) Case II

FIGURE 2: State evolvement of multiagent systems with two topology cases when $\beta_{0}=2$, and $\tau_{1}(t)=0.12 \mathrm{~s}$.

(1) The stability criteria can be obtained to determine the robust consensus through justifying the existence of common Lyapunov-Krasovskii functional.

(2) The existence of common Lyapunov-Krasovskii functional is equivalent to solving feasible solution of NLMI. In order to assure lower conservativeness than the existing one [19], FWM method was employed to justify whether the derivative of the functional is negative definite.

(3) Nonlinear minimization methods were used to convert the NLMI criteria into the problem which can be solved easily by the existing LMI-based approaches.

(4) Moreover, the proposed conclusions also characterized the relationship among communication delays, their derivative, and the coefficient of the consensus protocol for any given $\gamma$ index. It can be regarded as an important basis to depress the influence of limited communication conditions effectively.

\section{Appendix}

\section{Proof of Theorem 7}

By applying the idea of state decomposition and space transformation, the condition of multiagent systems achieving robust consensus is equivalent to the stability of the system (A.1); the detailed procedure can be seen in [8].

Consider

$$
\begin{gathered}
\dot{\delta}_{2}^{\dagger}(t)=-\beta_{0} \sum_{q=1}^{m}\left(\widetilde{\mathbf{L}}_{\sigma q}+\Delta \widetilde{\mathbf{L}}_{\sigma q}\right) \delta_{2}^{\dagger}\left(t-\tau_{q}\right)+\omega_{2}^{\dagger}(t), \\
\mathbf{z}_{2}^{\dagger}(t)=\widetilde{\Lambda} \delta_{2}^{\dagger}(t),
\end{gathered}
$$

where $\boldsymbol{\delta}(t)$ denotes the disagreement systems according to state decomposition idea. If disagreement variable $\delta(t) \rightarrow 0$ when $t \rightarrow \infty$, this means that state variable $\mathbf{x}(t)$ converges to the average value of its initial value. The new variable $\widetilde{\delta}=\mathbf{E}_{c}^{T} \delta=\left[\widetilde{\delta}_{1}^{\dagger}, \widetilde{\delta}_{2}^{\dagger}\right]$ can be obtained by space transformation method; its definition can be depicted as follows:

$$
\begin{gathered}
\widetilde{\delta}_{1}^{\dagger}=\left[\widetilde{\delta}_{1}, \ldots, \widetilde{\delta}_{n-\kappa}\right]^{T}, \\
\widetilde{\delta}_{2}^{\dagger}=\left[\widetilde{\delta}_{n-\kappa+1}, \ldots, \widetilde{\delta}_{n}\right]^{T},
\end{gathered}
$$

where $\kappa$ is the rank of $\sum_{i=1}^{m} \mathbf{L}_{\sigma i}$. Matrix $\mathbf{E}_{c}$ is composed of the eigenvectors of the Laplacian matrix of complete graph. Moreover, $\mathbf{E}_{c \kappa}$ is the former $\kappa$ columns of the aforementioned matrix $\mathbf{E}_{c}$. Then we have

$$
\begin{aligned}
\tilde{\mathbf{L}}_{\sigma q} & =\mathbf{E}_{c \kappa}^{T} \mathbf{L}_{\sigma q} \mathbf{E}_{c \kappa}, \\
\Delta \tilde{\mathbf{L}}_{\sigma q} & =\mathbf{E}_{c \kappa}^{T} \Delta \mathbf{L}_{\sigma q} \mathbf{E}_{c \kappa}, \\
\widetilde{\Lambda} & =\mathbf{E}_{c \kappa}^{T} \Lambda \mathbf{E}_{c \kappa} .
\end{aligned}
$$

If multiagent systems achieve robust consensus for specified $H_{\infty}$ index $\gamma$, two conditions should be satisfied:

(C1) $\forall t \geq 0$, systems (A.1) are asymptotically stable under the condition $\omega_{2}^{\dagger}(t)=0$

(C2) $\mathbf{J}\left(\boldsymbol{\omega}_{2}^{\dagger}\right)<0$ for all nonzero $\boldsymbol{\omega}_{2}^{\dagger}(t) \in \mathscr{L}_{2}[0, \infty)$ and specified $\gamma>0$, where $\mathbf{J}\left(\boldsymbol{\omega}_{2}^{\dagger}\right)=\int_{0}^{\infty}\left(\left[\mathbf{z}_{2}^{\dagger}(t)\right]^{T} \mathbf{z}_{2}^{\dagger}(t)-\right.$ $\left.\gamma^{2}\left[\omega_{2}^{\dagger}(t)\right]^{T} \boldsymbol{\omega}_{2}^{\dagger}(t)\right) d t$

In the following, the detailed process will be given to prove the stability of the system (A.1). 


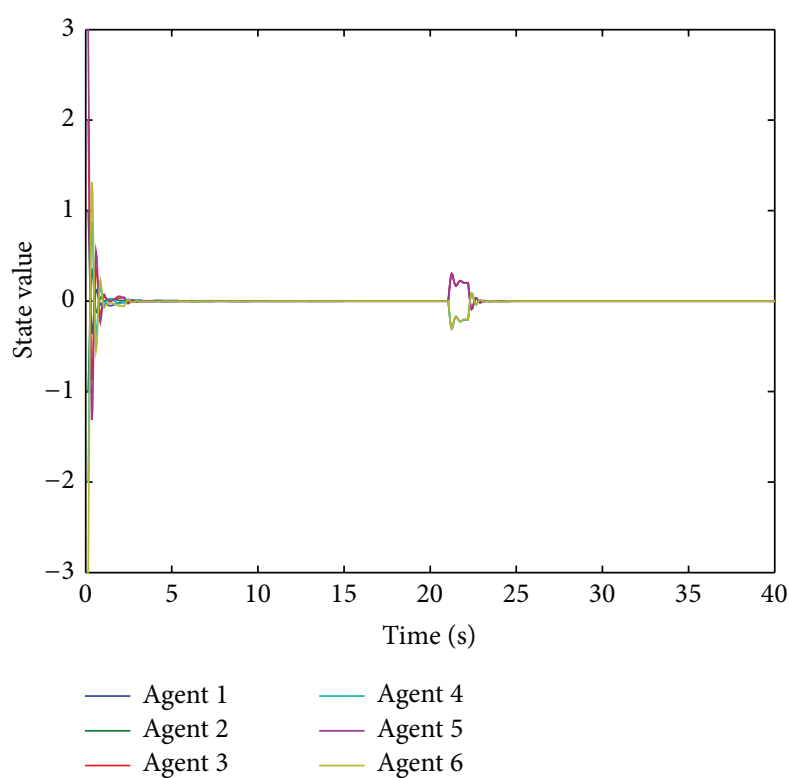

(a) Case I: $\tau_{1}(t)=0.2 \mathrm{~s}$

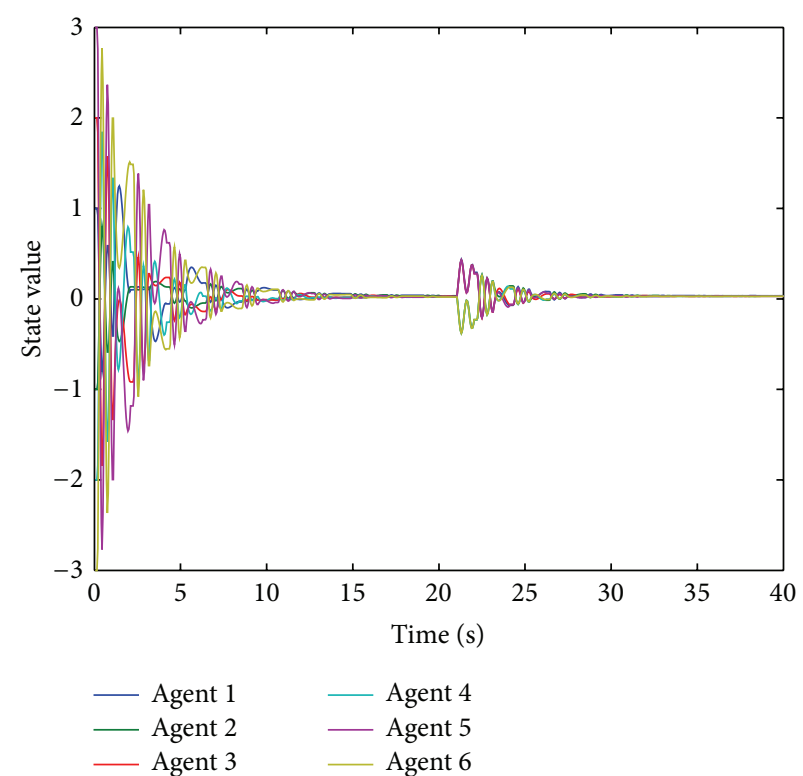

(b) Case I: $\tau_{1}(t)=0.3 \mathrm{~s}$

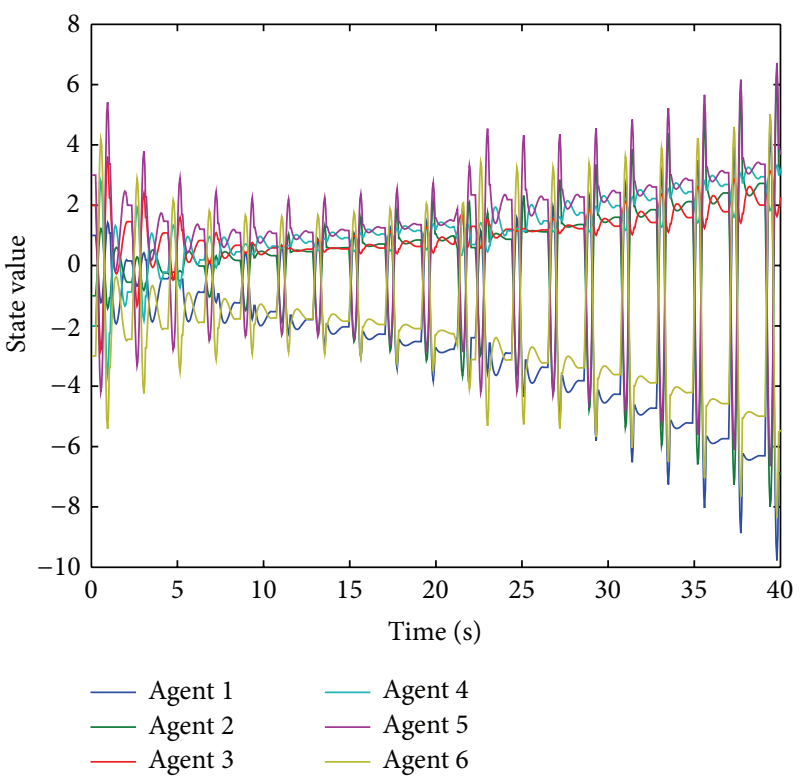

(c) Case I: $\tau_{1}(t)=0.4 \mathrm{~s}$

FIGURE 3: State evolvement of multiagent systems with different $\tau_{1}(t)$ when $\beta_{0}=2$ and $\tau_{2}(t)=0.917|\sin ((0.5 / 0.917) t)| \mathrm{s}$.

Proof. Considering all jointly connected topologies determined by $\sigma:[0, \infty] \rightarrow\left\{1,2, \ldots, N_{\text {ss }}\right\}$, define the following common jointly quadratic Lyapunov-Krasovskii (CJQLK) functional:

$$
\begin{aligned}
\mathbf{V}\left(t, \boldsymbol{\delta}_{2}^{\dagger}(t)\right)= & {\left[\boldsymbol{\delta}_{2}^{\dagger}(t)\right]^{T} \mathbf{P} \boldsymbol{\delta}_{2}^{\dagger}(t) } \\
& +\sum_{q=1}^{m} \int_{t-\tau_{q}}^{t}\left[\boldsymbol{\delta}_{2}^{\dagger}(s)\right]^{T} \mathbf{Q}_{q} \boldsymbol{\delta}_{2}^{\dagger}(s) d s \\
& +\sum_{q=1}^{m} \int_{-\bar{\tau}_{q}}^{0} \int_{t+9}^{t}\left[\dot{\boldsymbol{\delta}}_{2}^{\dagger}(s)\right]^{T} \mathbf{R}_{q} \dot{\boldsymbol{\delta}}_{2}^{\dagger}(s) d s d \vartheta
\end{aligned}
$$

where $\mathbf{P}=\mathbf{P}^{T}>0, \mathbf{Q}_{q}=\mathbf{Q}_{q}^{T} \geq 0$, and $\mathbf{R}_{q}=\mathbf{R}_{q}^{T} \geq$ $0, q=1,2, \ldots, m$ are common matrices with appropriate dimensions.

Given free-weighting matrices

$$
\mathbf{N}_{q}=\left[\mathbf{N}_{q}^{1}, \mathbf{N}_{q}^{2}\right]^{T}, \quad q=1,2, \ldots, m,
$$

and any matrices with proper dimensions

$$
\mathbf{M}_{q}=\left[\begin{array}{cc}
\mathbf{M}_{q}^{11} & \mathbf{M}_{q}^{12} \\
\star & \mathbf{M}_{q}^{22}
\end{array}\right] \geq 0, \quad q=1,2, \ldots, m,
$$


by Newton-Leibniz formula, we have

$$
\begin{aligned}
& \sum_{q=1}^{m} 2\left\{\left[\boldsymbol{\delta}_{2}^{\dagger}(t)\right]^{T} \mathbf{N}_{q}^{1}+\left[\boldsymbol{\delta}_{2}^{\dagger}\left(t-\tau_{q}\right)\right]^{T} \mathbf{N}_{q}^{2}\right\} \\
& \times\left[\boldsymbol{\delta}_{2}^{\dagger}(t)-\boldsymbol{\delta}_{2}^{\dagger}\left(t-\tau_{q}\right)-\int_{t-\tau_{q}}^{t} \dot{\boldsymbol{\delta}}_{2}^{\dagger}(s) d s\right]=0 \\
& \sum_{q=1}^{m}\left[\tau_{q} \xi_{1 q}^{T}(t) \mathbf{M}_{q} \boldsymbol{\xi}_{1 q}(t)-\int_{t-\tau_{q}}^{t} \boldsymbol{\xi}_{1 q}^{T}(t) \mathbf{M}_{q} \boldsymbol{\xi}_{1 q}(t) d s\right]=0
\end{aligned}
$$

where $\boldsymbol{\xi}_{1 q}(t)=\left[\left[\boldsymbol{\delta}_{2}^{\dagger}(t)\right]^{T},\left[\boldsymbol{\delta}_{2}^{\dagger}\left(t-\tau_{q}\right)\right]^{T}\right]^{T}$.

Calculating the derivative of $V\left(t, \boldsymbol{\delta}_{2}^{\dagger}(t)\right)$,

$$
\begin{aligned}
\dot{\mathbf{V}}\left(t, \delta_{2}^{\dagger}(t)\right)= & 2\left[\delta_{2}^{\dagger}(t)\right]^{T} \mathbf{P} \dot{\delta}_{2}^{\dagger}(t)+\sum_{q=1}^{m}\left[\delta_{2}^{\dagger}(t)\right]^{T} \mathbf{Q}_{q} \delta_{2}^{\dagger}(t) \\
& -\sum_{q=1}^{m}\left(1-\dot{\tau}_{q}\right)\left[\delta_{2}^{\dagger}\left(t-\tau_{q}\right)\right]^{T} \mathbf{Q}_{q} \delta_{2}^{\dagger}\left(t-\tau_{q}\right) \\
& +\sum_{q=1}^{m} \bar{\tau}_{q}\left[\dot{\delta}_{2}^{\dagger}(t)\right]^{T} \mathbf{R}_{q} \dot{\delta}_{2}^{\dagger}(t) \\
& -\sum_{q=1}^{m} \int_{t-\bar{\tau}_{q}}^{t}\left[\dot{\delta}_{2}^{\dagger}(s)\right]^{T} \mathbf{R}_{q} \dot{\delta}_{2}^{\dagger}(s) d s .
\end{aligned}
$$

Denote $\digamma_{1}=-\beta_{0}\left(\widetilde{\mathbf{L}}_{\sigma q}+\Delta \widetilde{\mathbf{L}}_{\sigma q}\right) \delta_{2}^{\dagger}\left(t-\tau_{q}\right)+\omega_{2}(t)$ and $\digamma_{2}$ and $F_{3}$ equals to the left terms of (A.7) and (A.8), respectively. Substituting $\dot{\boldsymbol{\delta}}_{2}^{\dagger}(t)$ with the expression in (A.1) and adding $F_{2}$ and $F_{3}$ yield

$$
\begin{aligned}
\dot{\mathbf{V}}(t, & \left.\boldsymbol{\delta}_{2}^{\dagger}(t)\right) \\
\leq & -2 \beta_{0}\left[\boldsymbol{\delta}_{2}^{\dagger}(t)\right]^{T} \mathbf{P} \sum_{q=1}^{m}\left(\widetilde{\mathbf{L}}_{\sigma q}+\Delta \widetilde{\mathbf{L}}_{\sigma q}\right)\left[\boldsymbol{\delta}_{2}^{\dagger}\left(t-\tau_{q}\right)\right]^{T} \\
& +2\left[\boldsymbol{\delta}_{2}^{\dagger}(t)\right]^{T} \mathbf{P} \boldsymbol{\omega}_{2}(t)+\sum_{q=1}^{m}\left[\boldsymbol{\delta}_{2}^{\dagger}(t)\right]^{T} \mathbf{Q}_{q} \boldsymbol{\delta}_{2}^{\dagger}(t) \\
& -\sum_{q=1}^{m}\left(1-\mu_{q}\right)\left[\boldsymbol{\delta}_{2}^{\dagger}\left(t-\tau_{q}\right)\right]^{T} \mathbf{Q}_{q} \boldsymbol{\delta}_{2}^{\dagger}\left(t-\tau_{q}\right) \\
& +\sum_{q=1}^{m} \bar{\tau}_{q} F_{1}^{T} \mathbf{R}_{q} F_{1}+F_{2}+F_{3} .
\end{aligned}
$$

The proof procedure of $(\mathrm{C} 1)$, that is, the stability of system (A.1) under nonzero $\omega_{2}^{\dagger}(t) \in \mathscr{L}_{2}[0, \infty)$ condition and specified $H_{\infty}$ index $\gamma>0$.

Rearrange the inequality (A.10) when $\omega_{2}^{\dagger}(t) \neq 0$.
Consider

$$
\begin{aligned}
\dot{\mathbf{V}}(t, & \left.\boldsymbol{\delta}_{2}^{\dagger}(t)\right) \\
\leq & \sum_{q=1}^{m} \boldsymbol{\xi}_{2 q}^{T}(t) \boldsymbol{\Xi}_{2 q} \boldsymbol{\xi}_{2 q}(t)+\gamma^{2}\left[\boldsymbol{\omega}_{2}^{\dagger}(t)\right]^{T} \boldsymbol{\omega}_{2}^{\dagger}(t) \\
& -\sum_{q=1}^{m} \int_{t-\tau_{q}}^{t} \boldsymbol{\xi}_{3 q}^{T}(t, s) \boldsymbol{\Xi}_{3 q} \boldsymbol{\xi}_{3 q}(t, s) d s-\left[\mathbf{z}_{2}^{\dagger}(t)\right]^{T} \mathbf{z}_{2}^{\dagger}(t),
\end{aligned}
$$

where

$$
\begin{aligned}
& \boldsymbol{\xi}_{2 q}(t)=\left[\boldsymbol{\xi}_{1 q}(t),\left[\boldsymbol{\omega}_{2}^{\dagger}(t)\right]^{T}\right]^{T} \\
& \boldsymbol{\xi}_{3 q}(t, s)=\left[\boldsymbol{\xi}_{1 q}(t),\left[\dot{\boldsymbol{\delta}}_{2}^{\dagger}(s)\right]^{T}\right]^{T} \\
& \boldsymbol{\Xi}_{2 q}=\left[\begin{array}{ccc}
\boldsymbol{\Xi}_{2 q}^{11} & \boldsymbol{\Xi}_{2 q}^{12} & \mathbf{P} \\
\star & \boldsymbol{\Xi}_{2 q}^{22} & 0 \\
\star & \star & -\gamma^{2} \mathbf{I}
\end{array}\right]+[\widetilde{\boldsymbol{\Lambda}}, 0,0]^{T}[\widetilde{\boldsymbol{\Lambda}}, 0,0] \\
& +\bar{\tau}_{q}\left[0,-\beta_{0}\left(\tilde{\mathbf{L}}_{\sigma q}+\Delta \tilde{\mathbf{L}}_{\sigma q}\right), 1\right]^{T} \mathbf{R}_{q} \\
& \times\left[0,-\beta_{0}\left(\widetilde{\mathbf{L}}_{\sigma q}+\Delta \tilde{\mathbf{L}}_{\sigma q}\right), 1\right] \\
& \boldsymbol{\Xi}_{2 q}^{11}=\mathbf{Q}_{q}+\mathbf{N}_{q}^{1}+\left[\mathbf{N}_{q}^{1}\right]^{T}+\bar{\tau}_{q} \mathbf{M}_{q}^{11} \\
& \boldsymbol{\Xi}_{2 q}^{12}=-\beta_{0} \mathbf{P}\left(\widetilde{\mathbf{L}}_{\sigma q}+\Delta \widetilde{\mathbf{L}}_{\sigma q}\right)-\mathbf{N}_{q}^{1}+\left[\mathbf{N}_{q}^{2}\right]^{T}+\bar{\tau}_{q} \mathbf{M}_{q}^{12} \\
& \mathbf{\Xi}_{2 q}^{22}=\left(\mu_{q}-1\right) \mathbf{Q}_{q}-\mathbf{N}_{q}^{2}-\left[\mathbf{N}_{q}^{2}\right]^{T}+\bar{\tau}_{q} \mathbf{M}_{q}^{22} \\
& \boldsymbol{\Xi}_{3 q}=\left[\begin{array}{ccc}
\mathbf{M}_{q}^{11} & \mathbf{M}_{q}^{12} & \mathbf{N}_{q}^{1} \\
\star & \mathbf{M}_{q}^{22} & \mathbf{N}_{q}^{2} \\
\star & \star & \mathbf{R}_{q}
\end{array}\right] \text {. }
\end{aligned}
$$

If $\sum_{q=1}^{m} \boldsymbol{\Xi}_{2 q}<0$ and $\sum_{q=1}^{m} \boldsymbol{\Xi}_{3 q}>0$ hold, then $\dot{\mathbf{V}}\left(t, \boldsymbol{\delta}_{2}^{\dagger}(t)\right)+$ $\left[\mathbf{z}_{2}^{\dagger}(t)\right]^{T} \mathbf{z}_{2}^{\dagger}(t)-\gamma^{2}\left[\boldsymbol{\omega}_{2}^{\dagger}(t)\right]^{T} \boldsymbol{\omega}_{2}^{\dagger}(t)<0$. Calculating the integral over $[0, \infty)$ with initial value $\mathbf{V}(0)=0$, we have

$$
\int_{0}^{\infty} \dot{\mathbf{V}}\left(t, \boldsymbol{\delta}_{2}^{\dagger}(t)\right)+\left[\mathbf{z}_{2}^{\dagger}(t)\right]^{T} \mathbf{z}_{2}^{\dagger}(t)-\gamma^{2}\left[\boldsymbol{\omega}_{2}^{\dagger}(t)\right]^{T} \boldsymbol{\omega}_{2}^{\dagger}(t) d t<0
$$

or compactly $\left\|\mathbf{z}_{2}^{\dagger}(t)\right\|_{2}<\gamma\left\|\boldsymbol{\omega}_{2}^{\dagger}(t)\right\|_{2}$.

Substitute $\Delta \mathbf{L}_{\sigma q}$ in $\boldsymbol{\Xi}_{2 q}$ with (8). Considering $\forall q \in[1, m]$, we have the following equivalent relationship according to Lemmas 4 and 5:

$$
\begin{aligned}
\boldsymbol{\Xi}_{2 q}<0, & \boldsymbol{\Xi}_{3 q}>0 \\
\sim & {\left[\begin{array}{cccc}
\boldsymbol{\Xi}_{2 q a} & \boldsymbol{\Xi}_{2 q b} & \boldsymbol{\Xi}_{2 q c} & \boldsymbol{\Xi}_{2 q d} \\
\star & -\bar{\tau}_{q} \mathbf{R}_{q}^{-1} & 0 & 0 \\
\star & \star & -\bar{\tau}_{q} \mathbf{R}_{q} & 0 \\
\star & \star & \star & -\mathbf{I}
\end{array}\right]<0, }
\end{aligned}
$$




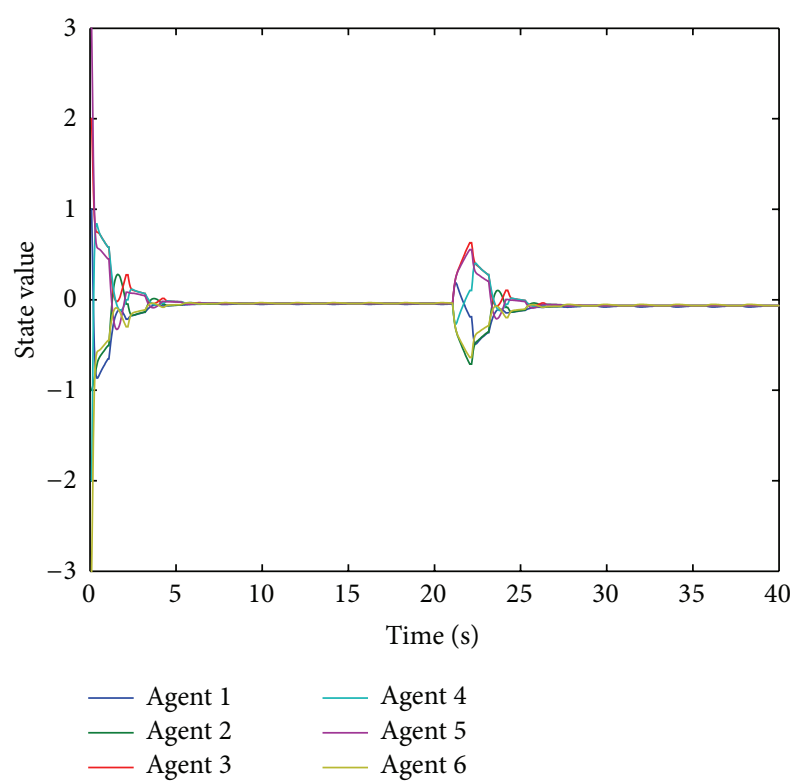

(a) Case II: $\beta_{0}=1$

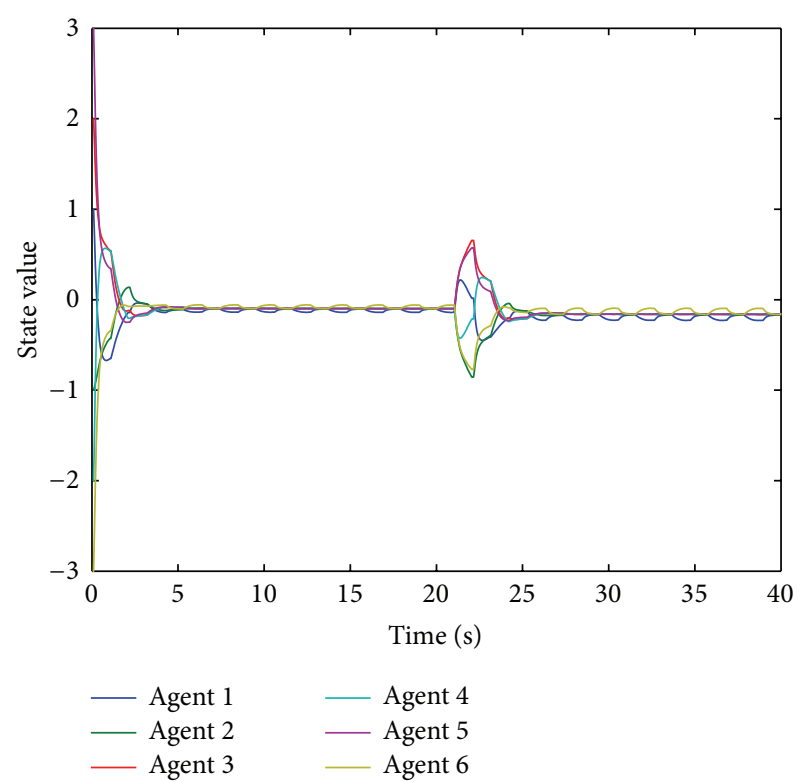

(b) Case II: $\beta_{0}=0.5$

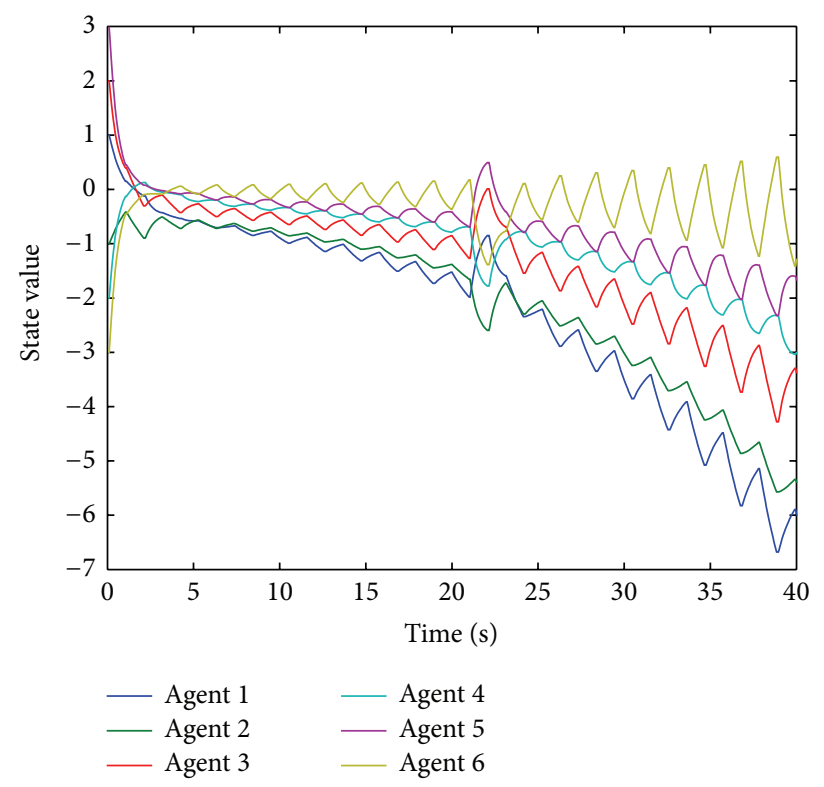

(c) Case II: $\beta_{0}=0.05$

FIGURE 4: State evolvement of multiagent systems with different $\beta_{0}$ when $\tau_{1}(t)=0.12 \mathrm{~s}$ and $\tau_{2}(t)=5.197|\sin ((0.5 / 5.197) t)| \mathrm{s}$.

where

$$
\begin{gathered}
\Xi_{2 q a}=\left[\begin{array}{ccc}
\Xi_{2 q a}^{11} & \Xi_{2 q a}^{12} & \mathbf{P} \\
\star & \Xi_{2 q a}^{22} & 0 \\
\star & \star & -\gamma^{2} \mathbf{I}
\end{array}\right] \\
\Xi_{2 q a}^{11}=\Xi_{2 q}^{11}-\bar{\tau}_{q} \mathbf{M}_{q}^{11} \\
\Xi_{2 q a}^{12}=\Xi_{2 q}^{12}-\bar{\tau}_{q} \mathbf{M}_{q}^{12} \\
\Xi_{2 q a}^{22}=\Xi_{2 q}^{22}-\bar{\tau}_{q} \mathbf{M}_{q}^{22}
\end{gathered}
$$

Moreover, inequality (A.14) is reformulated as follows

$$
\boldsymbol{\Phi}_{q}+\Psi_{q_{1}} \boldsymbol{\Omega}_{\sigma}(t) \Psi_{q_{2}}^{T}+\Psi_{q_{2}} \boldsymbol{\Omega}_{\sigma}^{T}(t) \Psi_{q_{1}}^{T}<0
$$




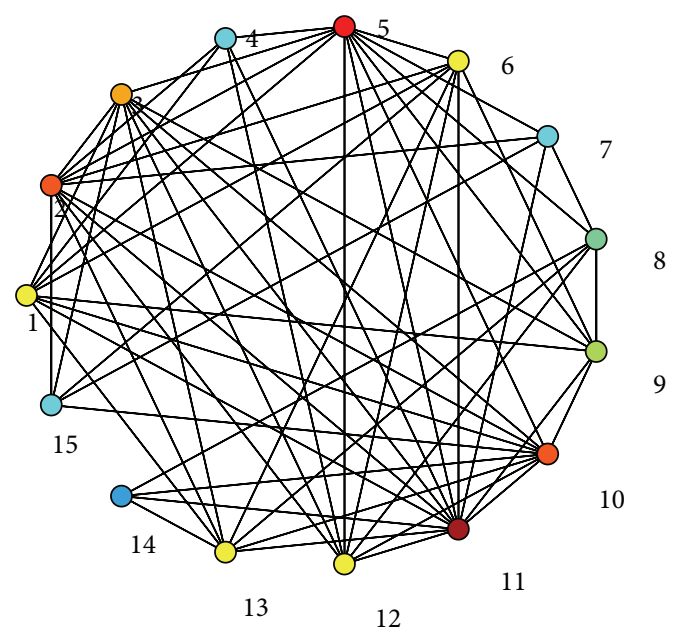

FIGURE 5: Strongly-connected and balanced graph $G_{e}$ with 15 nodes.

Here,

$$
\begin{aligned}
& \boldsymbol{\Phi}_{q}=\left[\begin{array}{cccccc}
\boldsymbol{\Phi}_{q}^{11} & \boldsymbol{\Phi}_{q}^{12} & \mathbf{P} & 0 & \bar{\tau}_{q} \mathbf{N}_{q}^{1} & \bar{\tau}_{q} \tilde{\boldsymbol{\Lambda}}^{T} \\
\star & \boldsymbol{\Phi}_{q}^{22} & 0 & \boldsymbol{\Phi}_{q}^{24} & \bar{\tau}_{q} \mathbf{N}_{q}^{2} & 0 \\
\star & \star & -\gamma^{2} \mathbf{I} & \bar{\tau}_{q} & 0 & 0 \\
\star & \star & \star & \boldsymbol{\Phi}_{q}^{44} & 0 & 0 \\
\star & \star & \star & \star & \boldsymbol{\Phi}_{q}^{55} & 0 \\
\star & \star & \star & \star & \star & -\mathbf{I}
\end{array}\right], \\
& \Phi_{q}^{11}=\Xi_{2 q a}^{11}, \quad \Phi_{q}^{12}=\Xi_{2 q a}^{12}+\beta_{0} \mathbf{P} \Delta \widetilde{\mathbf{L}}_{\sigma q}, \quad \Phi_{q}^{22}=\Xi_{2 q a}^{22}, \\
& \boldsymbol{\Phi}_{q}^{24}=-\bar{\tau}_{q} \beta_{0} \widetilde{\mathbf{L}}_{\sigma q}, \quad \boldsymbol{\Phi}_{q}^{44}=-\bar{\tau}_{q} \mathbf{R}_{q}^{-1}, \quad \boldsymbol{\Phi}_{q}^{55}=-\bar{\tau}_{q} \mathbf{R}_{q}, \\
& \Psi_{q_{1}}=\left[-\beta_{0} \mathbf{P} \widetilde{\mathbf{L}}_{\sigma q}^{1}, 0,0,-\beta_{0} \bar{\tau}_{q} \widetilde{\mathbf{L}}_{\sigma q}^{1}, 0,0\right]^{T}, \\
& \Psi_{q_{2}}=\left[0,\left[\widetilde{\mathbf{L}}_{\sigma q}^{2}\right]^{T}, 0,0,0,0\right]^{T} .
\end{aligned}
$$

According to Lemma 6, we have

$$
\boldsymbol{\Phi}_{q}+c_{0}^{-1} \Psi_{q_{1}} \Psi_{q_{1}}^{T}+c_{0} \Psi_{q_{2}} \boldsymbol{\Psi}_{q_{2}}^{T}<0 \sim \boldsymbol{\Xi}_{q}<0
$$

If $\sum_{q=1}^{m} \Xi_{q}<0$, then the condition (A.13) holds. This means that the multiagent systems are stable with nonzero $\omega_{2}^{\dagger}(t) \in \mathscr{L}_{2}[0, \infty)$ and a given $\gamma>0$. The proof procedure of (C2), that is, the stability of system (A.1) under the condition $\boldsymbol{\omega}_{2}^{\dagger}(t)=0$.

Rearrange (A.10) when $\omega_{2}^{\dagger}(t)=0$.
Consider

$$
\begin{aligned}
\dot{\mathbf{V}}\left(t, \boldsymbol{\delta}_{2}^{\dagger}(t)\right) \leq & \sum_{q=1}^{m} \boldsymbol{\xi}_{1 q}^{T}(t) \boldsymbol{\Xi}_{1 q} \boldsymbol{\xi}_{1 q}(t) \\
& -\sum_{q=1}^{m} \int_{t-\tau_{q}(t)}^{t} \boldsymbol{\xi}_{3 q}^{T}(t, s) \boldsymbol{\Xi}_{3 q} \xi_{3 q}(t, s) d s,
\end{aligned}
$$

where $\boldsymbol{\Xi}_{1 q}=\left[\begin{array}{cc}\Phi_{q}^{11} & \Phi_{q}^{12} \\ \star & \Phi_{q}^{22}\end{array}\right]+\bar{\tau}_{q}\left[0,-\beta_{0}\left(\widetilde{\mathbf{L}}_{\sigma q}+\Delta \widetilde{\mathbf{L}}_{\sigma q}\right)\right]^{T} \times$ $\mathbf{R}_{q}\left[0,-\beta_{0}\left(\widetilde{\mathbf{L}}_{\sigma q}+\Delta \widetilde{\mathbf{L}}_{\sigma q}\right)\right]$ and $\boldsymbol{\Phi}_{q}^{11}, \boldsymbol{\Phi}_{q}^{12}, \boldsymbol{\Phi}_{q}^{22}, \boldsymbol{\xi}_{1 q}$, and $\boldsymbol{\xi}_{3 q}, \Xi_{q}^{3}$ are defined in (A.8), (A.11), and (A.16).

Similarly, if $\Xi_{1 q}<0$ and $\Xi_{3 q}>0$ holds, we get

$$
\left[\begin{array}{cccccc}
\boldsymbol{\Phi}_{q}^{11} & \boldsymbol{\Phi}_{q}^{12} & 0 & \bar{\tau}_{q} \mathbf{N}_{q}^{1} & \boldsymbol{\Phi}_{q}^{15} & 0 \\
\star & \boldsymbol{\Phi}_{q}^{22} & \boldsymbol{\Phi}_{q}^{23} & \bar{\tau}_{q} \mathbf{N}_{q}^{2} & 0 & c_{0}\left[\widetilde{\mathbf{L}}_{\sigma q}^{2}\right]^{T} \\
\star & \star & \boldsymbol{\Phi}_{q}^{44} & 0 & \boldsymbol{\Phi}_{q}^{35} & 0 \\
\star & \star & \star & \boldsymbol{\Phi}_{q}^{55} & 0 & 0 \\
\star & \star & \star & \star & -c_{0} \mathbf{I} & 0 \\
\star & \star & \star & \star & \star & -c_{0} \mathbf{I}
\end{array}\right]<0,
$$

where

$$
\boldsymbol{\Phi}_{q}^{15}=-\beta_{0} \mathbf{P} \widetilde{\mathbf{L}}_{\sigma q}^{1}, \quad \boldsymbol{\Phi}_{q}^{23}=-\beta_{0} \bar{\tau}_{q} \widetilde{\mathbf{L}}_{\sigma q}^{T}, \quad \boldsymbol{\Phi}_{q}^{35}=-\beta_{0} \bar{\tau}_{q} \widetilde{\mathbf{L}}_{\sigma q}^{1} .
$$

It is clear that (A.20) can be derived from $\Xi_{q}<0$. That means, if $\sum_{q=1}^{m} \boldsymbol{\Xi}_{q}<0$ holds, multiagent systems are stable under the condition $\boldsymbol{\omega}_{2}^{\dagger}(t)=0$. Therefore, we can conclude that systems (A.1) are robust stable satisfying specified $H_{\infty}$ index $\gamma$. As a result, the multiagent systems asymptotically achieve robust consensus. The proof of Theorem 7 is complete.

\section{Conflict of Interests}

The authors declare that there is no conflict of interests regarding the publication of this paper.

\section{Acknowledgments}

This work was supported by NSFC (61203355) and STDP (20130522108JH) funded by China government.

\section{References}

[1] R. Olfati-Saber and R. M. Murray, "Consensus problems in networks of agents with switching topology and time-delays," IEEE Transactions on Automatic Control, vol. 49, no. 9, pp. 15201533, 2004.

[2] P.-A. Bliman and G. Ferrari-Trecate, "Average consensus problems in networks of agents with delayed communications," Automatica, vol. 44, no. 8, pp. 1985-1995, 2008.

[3] Y.-P. Tian and C.-L. Liu, "Robust consensus of multi-agent systems with diverse input delays and asymmetric interconnection perturbations," Automatica, vol. 45, no. 5, pp. 1347-1353, 2009.

[4] W. Wang and J.-J. E. Slotine, "Contraction analysis of timedelayed communications and group cooperation," IEEE Transactions on Automatic Control, vol. 51, no. 4, pp. 712-717, 2006. 
[5] Y. G. Sun, L. Wang, and G. Xie, "Average consensus in networks of dynamic agents with switching topologies and multiple timevarying delays," Systems \& Control Letters, vol. 57, no. 2, pp. 175183, 2008.

[6] P. Lin and Y. Jia, "Average consensus in networks of multi-agents with both switching topology and coupling time-delay," Physica A, vol. 387, no. 1, pp. 303-313, 2008.

[7] P. Lin, Y. Jia, J. Du, and F. Yu, “Average consensus for networks of continuous-time agents with delayed information and jointlyconnected topologies," in Proceedings of the American Control Conference (ACC '09), pp. 3884-3889, June 2009.

[8] Q. Zhang, Y. Niu, L. Wang, L. Shen, and H. Zhu, "Average consensus seeking of high-order continuous-time multi-agent systems with multiple time-varying communication delays," International Journal of Control, Automation and Systems, vol. 9, no. 6, pp. 1209-1218, 2011.

[9] M. Cao, A. S. Morse, and B. D. O. Anderson, "Reaching an agreement using delayed information," in Proceedings of the 45th IEEE Conference on Decision and Control (CDC '06), pp. 33753380, December 2006.

[10] F. Xiao and L. Wang, "Consensus protocols for discrete-time multi-agent systems with time-varying delays," Automatica, vol. 44, no. 10, pp. 2577-2582, 2008.

[11] L. Moreau, "Stability of continuous-time distributed consensus algorithms," in Proceedings of the 43rd IEEE Conference on Decision and Control (CDC '04), pp. 3998-4003, December 2004.

[12] V. D. Blondel, J. M. Hendrickx, A. Olshevsky, and J. N. Tsitsiklis, "Convergence in multiagent coordination, consensus, and flocking," in Proceedings of the 44th IEEE Conference on Decision and Control, and the European Control Conference (CDC-ECC '05), pp. 2996-3000, December 2005.

[13] Y.-Z. Sun and J. Ruan, "Consensus problems of multi-agent systems with noise perturbation," Chinese Physics B, vol. 17, no. 11, pp. 4137-4141, 2008.

[14] L. Wang and Z. Liu, "Robust consensus of multi-agent systems with noise," Science in China F, vol. 52, no. 5, pp. 824-834, 2009.

[15] Y. Liu and Y. Jia, " $H_{\infty}$ consensus control of multi-agent systems with switching topology: a dynamic output feedback protocol," International Journal of Control, vol. 83, no. 3, pp. 527-537, 2010.

[16] Y. Liu and Y. Jia, "Consensus problem of high-order multi-agent systems with external disturbances: an $H_{\infty}$ analysis approach," International Journal of Robust and Nonlinear Control, vol. 20, no. 14, pp. 1579-1593, 2010.

[17] U. Münz, A. Papachristodoulou, and F. Allgöwer, "Delay robustness in consensus problems," Automatica, vol. 46, no. 8, pp. 1252-1265, 2010.

[18] U. Münz, A. Papachristodoulou, and F. Allgower, "Consensus in multi-agent systems with coupling delays and switching topology," IEEE Transactions on Automatic Control, vol. 56, no. 12, pp. 2976-2982, 2011.

[19] P. Lin, Y. Jia, and L. Li, "Distributed robust $H_{\infty}$ consensus control in directed networks of agents with time-delay," Systems \& Control Letters, vol. 57, no. 8, pp. 643-653, 2008.

[20] P. Lin and Y. Jia, "Robust $H_{\infty}$ consensus analysis of a class of second-order multi-agent systems with uncertainty," IET Control Theory and Applications, vol. 4, no. 3, pp. 487-498, 2010.

[21] J. Hu, "On robust consensus of multi-agent systems with communication delays," Kybernetika, vol. 45, no. 5, pp. 768-784, 2009.
[22] Y. Liu and Y. Jia, "Robust $H_{\infty}$ consensus control of uncertain multi-agent systems with time delays," International Journal of Control, Automation and Systems, vol. 9, no. 6, pp. 1086-1094, 2011.

[23] Y. Liu and Y. Jia, " $H_{\infty}$ consensus control for multi-agent systems with linear coupling dynamics and communication delays," International Journal of Systems Science, vol. 43, no. 1, pp. 5062, 2012.

[24] M. Wu, Y. He, J.-H. She, and G.-P. Liu, "Delay-dependent criteria for robust stability of time-varying delay systems," Automatica, vol. 40, no. 8, pp. 1435-1439, 2004.

[25] Y. He, M. Wu, J.-H. She, and G.-P. Liu, "Parameter-dependent Lyapunov functional for stability of time-delay systems with polytopic-type uncertainties," IEEE Transactions on Automatic Control, vol. 49, no. 5, pp. 828-832, 2004.

[26] Y. He, M. Wu, J.-H. She, and G.-P. Liu, "Delay-dependent robust stability criteria for uncertain neutral systems with mixed delays," Systems \& Control Letters, vol. 51, no. 1, pp. 57-65, 2004.

[27] A. Jadbabaie, J. Lin, and A. S. Morse, "Coordination of groups of mobile autonomous agents using nearest neighbor rules," in Proceedings of the 41st IEEE Conference on Decision and Control (CDC '02), pp. 2953-2958, December 2002.

[28] Y. M. Jia, Robust $H_{\infty}$ Control, Science Press, Beijing, China, 2007.

[29] K. Gu, "A further refinement of discretized Lyapunov functional method for the stability of time-delay systems," International Journal of Control, vol. 74, no. 10, pp. 967-976, 2001.

[30] I. R. Petersen and C. V. Hollot, "A riccati equation approach to the stabilization of uncertain linear systems," Automatica, vol. 22, no. 4, pp. 397-411, 1986.

[31] L. El Ghaoui, F. Oustry, and M. AitRami, "A cone complementarity linearization algorithm for static output-feedback and related problems," IEEE Transactions on Automatic Control, vol. 42, no. 8, pp. 1171-1176, 1997. 


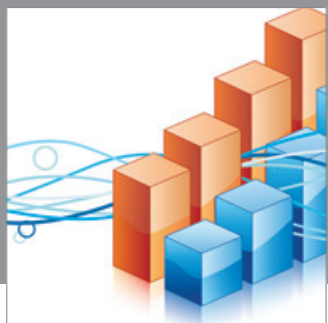

Advances in

Operations Research

mansans

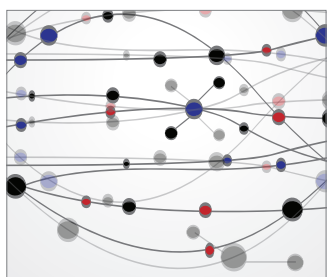

The Scientific World Journal
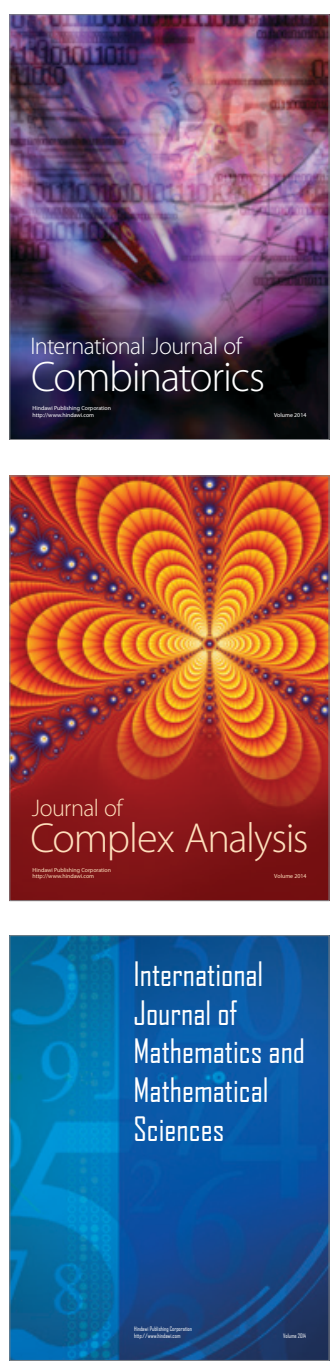
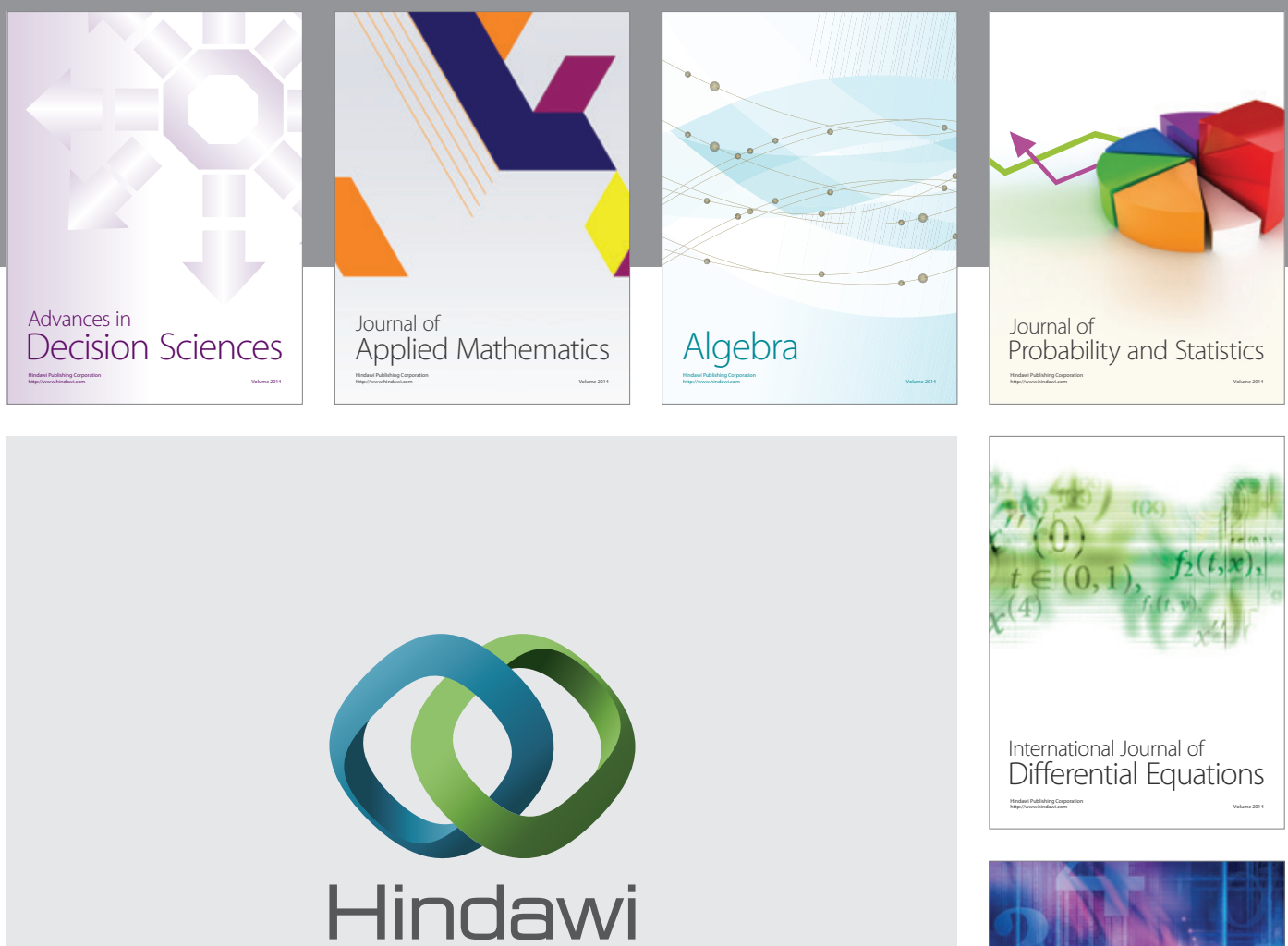

Submit your manuscripts at http://www.hindawi.com
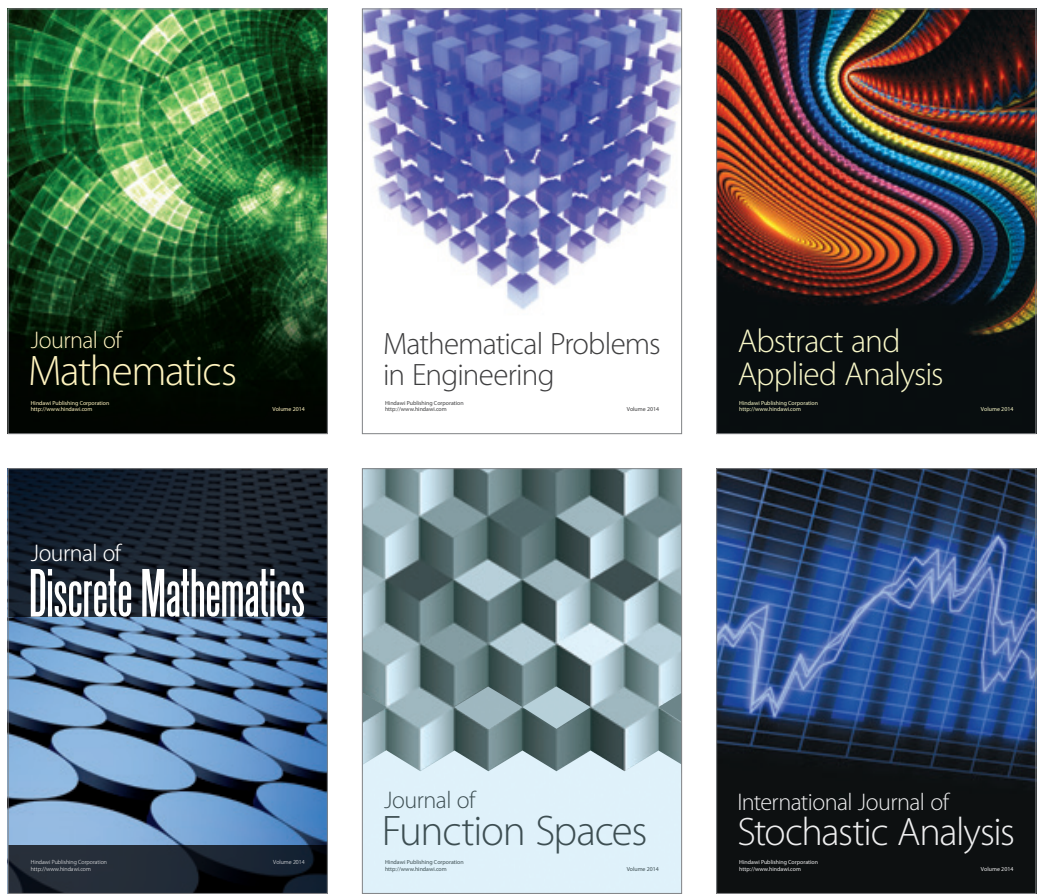

Journal of

Function Spaces

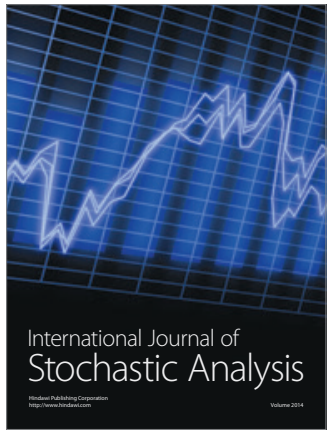

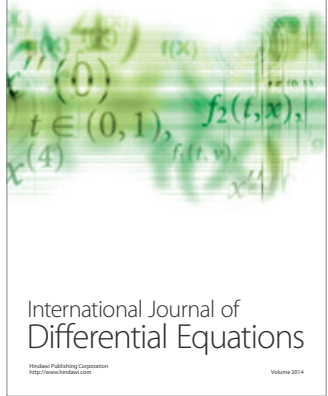
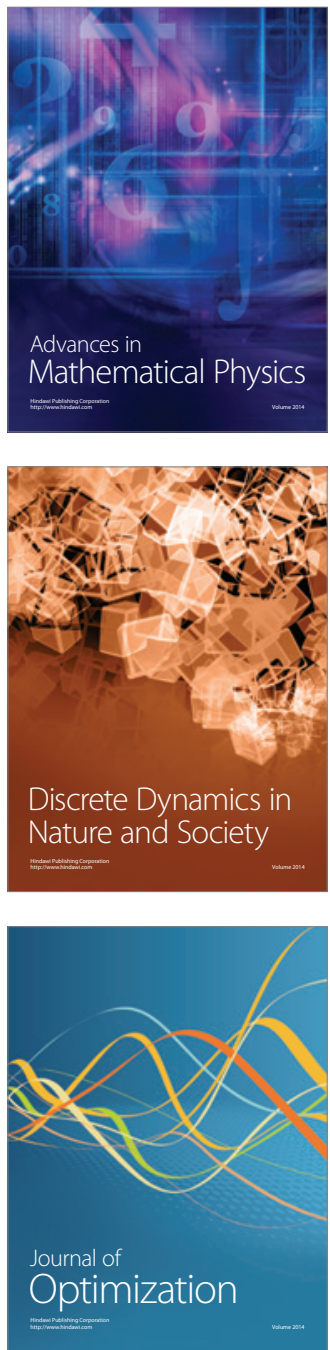